\title{
The Stokes Phenomenon for Some Moment Partial Differential Equations
}

\author{
Sławomir Michalik ${ }^{1}$ (D) . Bożena Tkacz ${ }^{1}$
}

Received: 9 June 2018 / Revised: 9 November 2018 / Published online: 5 December 2018

(C) The Author(s) 2018

\begin{abstract}
We study the Stokes phenomenon for the solutions of general homogeneous linear moment partial differential equations with constant coefficients in two complex variables under condition that the Cauchy data are holomorphic on the complex plane but finitely many singular or branching points with the appropriate growth condition at the infinity. The main tools are the theory of summability and multisummability, and the theory of hyperfunctions. Using them, we describe Stokes lines, anti-Stokes lines, jumps across Stokes lines, and a maximal family of solutions.
\end{abstract}

Keywords Linear PDEs with constant coefficients · Moment-PDEs · Borel summability · Multisummability $\cdot$ Maximal family of solutions $\cdot$ Stokes phenomenon $\cdot$ Hyperfunctions

Mathematics Subject Classification (2010) $35 \mathrm{C} 10 \cdot 35 \mathrm{C} 20 \cdot 35 \mathrm{E} 15 \cdot 40 \mathrm{G} 10$

\section{Introduction}

In this article, we generalise our results from [18] concerning summability and Stokes phenomenon for the formal solutions of the Cauchy problem for the complex heat equation. In the present paper, we consider the Cauchy problem for general homogeneous linear moment partial differential equation with constant coefficients in two complex variables $(t, z)$

$$
\left\{\begin{array}{l}
P\left(\partial_{m_{1}, t}, \partial_{m_{2}, z}\right) u=0 \\
\partial_{m_{1}, t}^{j} u(0, z)=\varphi_{j}(z) \in \mathcal{O}(D), j=0, \ldots, N-1,
\end{array}\right.
$$

where $P(\lambda, \zeta)$ is a polynomial of two variables of degree $N$ with respect to $\lambda$. Here, $\partial_{m_{1}, t}$ and $\partial_{m_{2}, z}$ denote the formal moment differentiations introduced by W. Balser and M. Yoshino [3], which generalise the usual and fractional differentiations.

Sławomir Michalik

s.michalik@uksw.edu.pl

http://www.impan.pl/ slawek

Bożena Tkacz

bpodhajecka@o2.pl

1 Faculty of Mathematics and Natural Sciences, College of Science, Cardinal Stefan Wyszyński University, Wóycickiego 1/3, 01-938 Warszawa, Poland 
Such type of equations was previously investigated by the first author [15-17] and by A. Lastra, S. Malek, and J. Sanz [11], mainly in the context of multisummability in a given direction.

Now, we use the similar methods as in the abovementioned papers to the study of multisummable normalised formal solution $\widehat{u}$ of Eq. 1 . It means that $\widehat{u}$ has to be multisummable in every direction but finitely many singular directions. For this reason, we assume that the Cauchy data have finitely many singular or branching points $z_{0}, \ldots, z_{n} \in \mathbb{C} \backslash\{0\}$ and are analytically continued to $\mathbb{C} \backslash \bigcup_{j=0}^{n}\left\{z_{j} t: t \geq 1\right\}$, and that satisfy the appropriate exponential growth condition at the infinity. Observe that by the linearity of Eq. 1, it is sufficient to consider the case when there is exactly one such point, say $z_{0} \in \mathbb{C} \backslash\{0\}$. Therefore, we only consider the case when $\varphi_{j}(z) \in \mathcal{O}\left(\widetilde{\mathbb{C} \backslash\left\{z_{0}\right\}}\right)$.

Using such formal multisummable solution $\widehat{u}$, for any nonsingular admissible multidirection $\mathbf{d}$, we are able to construct its multisum $u^{\mathbf{d}}$. This multisum is an actual solution of Eq. 1 as a holomorphic function in some sectorial neighbourhood of the origin.

The main purpose of this article is the description of these actual solutions and the study of the relations between them. To this end, we introduce the concept of maximal family of solutions. It is defined as the whole family of actual solutions, which can be obtained by the method of multisummability.

The relations between solutions are studied in the context of the Stokes phenomenon. It means that we find the Stokes lines, which separate different actual solutions constructed from the same multisummable formal power series solution. We also calculate the differences between actual solutions on such lines, which are called jumps across the Stokes lines. To study such jumps, we apply the Laplace-type hyperfunctions supported on the Stokes line.

In this way, we get the main result of the paper about the maximal family of solutions and the Stokes phenomenon for Eq. 1, which is given in Theorem 3.

In the special case when $\partial_{m_{1}, t}$ and $\partial_{m_{2}, z}$ are replaced by $\partial_{t}$ and $\partial_{z}$, we get the description of the Stokes phenomenon for general linear PDEs with constant coefficients.

In this sense, the paper gives the application of theory of summability for PDEs to the description of maximal family of solutions and to the study of Stokes phenomenon for such equations.

Let us recall that the theory of summability of the formal solutions of PDEs has been recently intensively developed by such authors as M. Hibino [4]; K. Ichinobe and M. Miyake [7]; K. Ichinobe [5, 6]; A. Lastra, S. Malek and J. Sanz [12]; P. Remy [19], H. Tahara and H. Yamazawa [21]; H. Yamazawa and M. Yoshino [23]; M. Yoshino [24, 25]; and others.

The paper is organised as follows. Section 2 consists of basic notations. In Section 3, we recall Balser's theory of moment summability. In particular, we introduce kernel functions and connected with them moment functions, Gevrey order, moment Borel and Laplace transforms, $k$-summability, and multisummability. In the next section, we recall the concept of moment differential operators and their generalisation to pseudodifferential operators. In Section 5, we recall the notion of Stokes phenomenon. We define Stokes lines and jumps across them for multisummable formal power series. We also introduce Laplace-type hyperfunction on Stokes lines, which allows us to describe these jumps. In Section 6, we introduce the idea of a maximal family of normalised actual solutions of non-Kowalevskian equation. We describe such family of solutions of Eq. 1 in the case when formal solution $\widehat{u}$ is multisummable (Theorem 1). In Section 7, we recall how to reduce the Cauchy problem (1) to a family of the Cauchy problems of simple pseudodifferential equations. Next, using the theory of moment summability, we find the integral representation of actual solutions of these 
simple pseudodifferential equations in the case when their formal solutions are summable (Proposition 5). It allows us to describe a maximal family of solutions of simple equations, Stokes lines, and jumps across them (Theorem 2). Finally, we return to the equation (1), and using the theory of multisummability, we get the main result of the paper, i.e. the description of a maximal family of solution, Stokes lines, and jumps across them for the equation (1), which is given in Theorem 3. In the last section, we present a few examples of special cases of moment partial differential equations with constant coefficients, where by using hyperfunctions we derive the form of jumps across obtained Stokes lines.

\section{Notation}

A sector $S$ in a direction $d \in \mathbb{R}$ with an opening $\alpha>0$ and a radius $R \in \mathbb{R}_{+}$in the universal covering space $\tilde{\mathbb{C}}$ of $\mathbb{C} \backslash\{0\}$ is defined by the following:

$$
S=S_{d}(\alpha, R)=\left\{z \in \tilde{\mathbb{C}}: z=r e^{i \phi}, r \in(0, R), \phi \in(d-\alpha / 2, d+\alpha / 2)\right\} .
$$

This sector is called unbounded if $R=+\infty$ and the notation $S=S_{d}(\alpha)$ will be used. If the opening $\alpha$ is not essential, the sector $S_{d}(\alpha)$ is denoted briefly by $S_{d}$.

A complex disc $D_{r}$ in $\mathbb{C}$ with a radius $r>0$ is a set of the form

$$
D_{r}=\{z \in \mathbb{C}:|z|<r\}
$$

In case that the radius $r$ is not essential, the set $D_{r}$ will be designated briefly by $D$. We also denote briefly a disc-sector $S_{d}(\alpha) \cup D$ (resp. $S_{d} \cup D$ ) by $\widehat{S}_{d}(\alpha)$ (resp. $\widehat{S}_{d}$ ).

If a function $f$ is holomorphic on a domain $G \subset \mathbb{C}^{n}$, then it will be denoted by $f \in \mathcal{O}(G)$. Analogously, the space of holomorphic functions of the variable $z^{1 / \gamma}=$ $\left(z_{1}^{1 / \gamma_{1}}, \ldots, z_{n}^{1 / \gamma_{n}}\right)$ on a domain $G \subset \mathbb{C}^{n}$ is denoted by $\mathcal{O}_{1 / \gamma}(G)$, where $z=\left(z_{1}, \ldots, z_{n}\right) \in$ $\mathbb{C}^{n}, \gamma=\left(\gamma_{1}, \ldots, \gamma_{n}\right) \in \mathbb{N}^{n}$ and $1 / \gamma=\left(1 / \gamma_{1}, \ldots, 1 / \gamma_{n}\right)$. In other words, $f \in \mathcal{O}_{1 / \gamma}(G)$ if and only if the function $w \mapsto f\left(w^{\gamma}\right)$ is analytic for every $w^{\gamma}=\left(w_{1}^{\gamma_{1}}, \ldots, w_{n}^{\gamma_{n}}\right) \in G$.

More generally, if $\mathbb{E}$ denotes a complex Banach space with a norm $\|\cdot\|_{\mathbb{E}}$, then by $\mathcal{O}(G, \mathbb{E})$ (resp. $\mathcal{O}_{1 / \gamma}(G, \mathbb{E})$ ), we shall denote the set of all $\mathbb{E}$-valued holomorphic functions (resp. holomorphic functions of the variables $z^{1 / \gamma}$ ) on a domain $G \subseteq \mathbb{C}^{n}$. For more information about functions with values in Banach spaces, we refer the reader to [2, Appendix B]. In the paper, as a Banach space $\mathbb{E}$, we will take the space of complex numbers $\mathbb{C}$ (we abbreviate $\mathcal{O}(G, \mathbb{C})$ to $\mathcal{O}(G)$ and $\mathcal{O}_{1 / \gamma}(G, \mathbb{C})$ to $\mathcal{O}_{1 / \gamma}(G)$ ) or the space of functions $E_{1 / \gamma}(D):=$ $\mathcal{O}_{1 / \gamma}(D) \cap C(\bar{D})$ equipped with the norm $\|\varphi\|_{E_{1 / \gamma}(D)}:=\max _{z \in \bar{D}}|\varphi(z)|$.

The space of formal power series $\sum_{n=0}^{\infty} a_{n} t^{n}$ with $a_{n} \in \mathbb{E}$ is denoted by $\mathbb{E}[[t]]$.

We use the "hat" notation $\left(\widehat{u}, \widehat{u}_{i}, \widehat{f}\right)$ to denote the formal power series. If the formal power series $\widehat{u}$ (resp. $\left.\widehat{u}_{i}, \widehat{f}\right)$ is convergent, we denote its sum by $u$ (resp. $u_{i}, f$ ).

Definition 1 Suppose $k \in \mathbb{R}, S$ is an unbounded sector and $u \in \mathcal{O}_{1 / \gamma}(S, \mathbb{E})$. The function $u$ is of exponential growth of order at most $k$, if for every proper subsector $S^{*} \prec S$ (i.e. $\overline{S^{*}} \backslash\{0\} \subseteq S$ ) there exist constants $C_{1}, C_{2}>0$ such that $\|u(x)\|_{\mathbb{E}} \leq C_{1} e^{C_{2}|x|^{k}}$ for every $x \in S^{*}$. If this is so, one can write $u \in \mathcal{O}_{1 / \gamma}^{k}(S, \mathbb{E})$ and $u \in \mathcal{O}_{1 / \gamma}^{k}(\mathbb{C}, \mathbb{E})$ for $S=\mathbb{C}$.

More generally, if $G$ is an unbounded domain in $\mathbb{C}^{n}$ and $u \in \mathcal{O}_{1 / \gamma}(G, \mathbb{E})$, then $u \in$ $\mathcal{O}_{1 / \gamma}^{k}(G, \mathbb{E})$ if for every set $G^{*}$ satisfying $\overline{G^{*}} \subset$ Int $G$ there exist constants $C_{1}, C_{2}>0$ such that $\|u(x)\|_{\mathbb{E}} \leq C_{1} e^{C_{2}|x|^{k}}$ for every $x \in G^{*}$. 


\section{Kernel and Moment Functions, K-Summability, and Multisummability}

In this section, we recall the notion of moment methods introduced by Balser [2]. It allows us to describe moment Borel transforms, Gevrey order, Borel summability, and multisummability.

Definition 2 (see [2, Section 5.5]) A pair of functions $e_{m}$ and $E_{m}$ is said to be kernel functions of order $k(k>1 / 2)$ if they have the following properties:

1. $e_{m} \in \mathcal{O}\left(S_{0}(\pi / k)\right), e_{m}(z) / z$ is integrable at the origin, $e_{m}(x) \in \mathbb{R}_{+}$for $x \in \mathbb{R}_{+}$and $e_{m}$ is exponentially flat of order $k$ as $z \rightarrow \infty$ in $S_{0}(\pi / k)$ (i.e., for every $\varepsilon>0$ there exist $A, B>0$ such that $\left|e_{m}(z)\right| \leq A e^{-(|z| / B)^{k}}$ for $\left.z \in S_{0}(\pi / k-\varepsilon)\right)$.

2. $E_{m} \in \mathcal{O}^{k}(\mathbb{C})$ and $E_{m}(1 / z) / z$ is integrable at the origin in $S_{\pi}(2 \pi-\pi / k)$.

3. The connection between $e_{m}$ and $E_{m}$ is given by the corresponding moment function $m$ of order $1 / k$ as follows. The function $m$ is defined by the Mellin transform of $e_{m}$

$$
m(u):=\int_{0}^{\infty} x^{u-1} e_{m}(x) d x \text { for } \operatorname{Re} u \geq 0
$$

and the kernel function $E_{m}$ has the power series expansion

$$
E_{m}(z)=\sum_{n=0}^{\infty} \frac{z^{n}}{m(n)} \quad \text { for } \quad z \in \mathbb{C} .
$$

4. Additionally, we assume that the corresponding moment function satisfies the normalisation property $m(0)=1$.

Remark 1 Observe that by the inverse Mellin transform and by Eq. 3, the moment function $m$ uniquely determines the kernel functions $e_{m}$ and $E_{m}$.

In case $k \leq 1 / 2$ the set $S_{\pi}(2 \pi-\pi / k)$ is not defined, so the second property in Definition 2 can not be satisfied. It means that we must define the kernel functions of order $k \leq 1 / 2$ and the corresponding moment functions in another way. To this end, we use the ramification at $z=0$.

Definition 3 (see [2, Section 5.6]) A function $e_{m}$ is called a kernel function of order $k>0$ if we can find a pair of kernel functions $e_{\tilde{m}}$ and $E_{\tilde{m}}$ of order $p k>1 / 2$ (for some $p \in \mathbb{N}$ ) so that

$$
e_{m}(z)=e_{\tilde{m}}\left(z^{1 / p}\right) / p \quad \text { for } \quad z \in S_{0}(\pi / k) .
$$

For a given kernel function $e_{m}$ of order $k>0$, we define the corresponding moment function $m$ of order $1 / k>0$ by Eq. 2 and the kernel function $E_{m}$ of order $k>0$ by Eq. 3 .

Remark 2 Observe that by Definitions 2 and 3 we have

$$
m(u)=\tilde{m}(p u) \quad \text { and } \quad E_{m}(z)=\sum_{j=0}^{\infty} \frac{z^{j}}{m(j)}=\sum_{j=0}^{\infty} \frac{z^{j}}{\tilde{m}(j p)} .
$$

As in [16], we extend the notion of moment functions to real orders. 
Definition 4 We say that $m$ is a moment function of order $1 / k<0$ if $1 / m$ is a moment function of order $-1 / k>0$.

We say that $m$ is a moment function of order 0 if there exist moment functions $m_{1}$ and $m_{2}$ of the same order $1 / k>0$ such that $m=m_{1} / m_{2}$.

By Definition 4 and by [2, Theorems 31 and 32] we have the following:

Proposition 1 Let $m_{1}, m_{2}$ be moment functions of orders $s_{1}, s_{2} \in \mathbb{R}$ respectively. Then we have as follows:

- $\quad m_{1} m_{2}$ is a moment function of order $s_{1}+s_{2}$.

- $m_{1} / m_{2}$ is a moment function of order $s_{1}-s_{2}$.

Example 1 For any $k>0$, the classical kernel functions and the corresponding moment function, satisfying Definition 2 or 3 , are given by the following:

- $e_{m}(z)=k z^{k} e^{-z^{k}}$,

- $m(u)=\Gamma(1+u / k)$,

- $E_{m}(z)=\sum_{j=0}^{\infty} \frac{z^{j}}{\Gamma(1+j / k)}=: \mathbf{E}_{1 / k}(z)$, where $\mathbf{E}_{1 / k}$ is the Mittag-Leffler function of index $1 / k$.

They are used in the classical theory of $k$-summability.

Example 2 For any $s \in \mathbb{R}$, we will denote by $\Gamma_{s}$ the function as follows:

$$
\Gamma_{s}(u):= \begin{cases}\Gamma(1+\mathrm{su}) & \text { for } s \geq 0 \\ 1 / \Gamma(1-\mathrm{su}) & \text { for } s<0 .\end{cases}
$$

Observe that by Example 1 and Definition $4, \Gamma_{s}$ is an example of a moment function of order $s \in \mathbb{R}$.

The moment functions $\Gamma_{s}$ will be extensively used in the paper, since every moment function $m$ of order $s$ has the same growth as $\Gamma_{s}$. Precisely speaking, we have the following:

Proposition 2 (see [2, Section 5.5]) If $m$ is a moment function of order $s \in \mathbb{R}$, then there exist constants $a, A, c, C>0$ such that

$$
a c^{n} \Gamma_{s}(n) \leq m(n) \leq A C^{n} \Gamma_{s}(n) \text { for every } n \in \mathbb{N}_{0} .
$$

Using Balser's theory of general moment summability ([2, Section 6.5], in particular [2, Theorem 38]), we apply the moment functions to define moment Borel transforms, the Gevrey order and the Borel summability. We first introduce the following:

Definition 5 Let $m$ be a moment function. Then, the linear operator $\widehat{\mathcal{B}}_{m}: \mathbb{E}[[t]] \rightarrow \mathbb{E}[[t]]$ defined as follows:

$$
\widehat{\mathcal{B}}_{m}\left(\sum_{j=0}^{\infty} u_{j} t^{j}\right):=\sum_{j=0}^{\infty} \frac{u_{j}}{m(j)} t^{j}
$$

is called an m-moment Borel transform. 
We define the Gevrey order of formal power series as follows:

Definition 6 Let $s \in \mathbb{R}$. Then, $\widehat{u} \in \mathbb{E}[[t]]$ is called a formal power series of Gevrey order $s$ if there exists a disc $D \subset \mathbb{C}$ with centre at the origin such that $\widehat{\mathcal{B}}_{\Gamma_{s}} \widehat{u} \in \mathcal{O}(D, \mathbb{E})$. The space of formal power series of Gevrey order $s$ is denoted by $\mathbb{E}[[t]]_{s}$.

Remark 3 By Proposition 2, we may replace $\Gamma_{s}$ in Definition 6 by any moment function $m$ of the same order $s$.

Remark 4 If $\widehat{u} \in \mathbb{E}[[t]]_{s}$ and $s \leq 0$ then the formal series $\widehat{u}$ is convergent, so its sum $u$ is well-defined. Moreover, $\widehat{u} \in \mathbb{E}[[t]]_{0} \Longleftrightarrow u \in \mathcal{O}(D, \mathbb{E})$ and $\widehat{u} \in \mathbb{E}[[t]]_{s} \Longleftrightarrow u \in$ $\mathcal{O}^{-1 / s}(\mathbb{C}, \mathbb{E})$ for $s<0$.

Definition 7 Let $e_{m}, E_{m}$ be a pair of kernel functions of order $1 / k>0$ with a moment function $m$ and let $d \in \mathbb{R}$.

- If $v \in \mathcal{O}^{k}\left(\widehat{S}_{d}, \mathbb{E}\right)$, then the integral operator $T_{m, d}$ defined as follows:

$$
\left(T_{m, d} v\right)(t):=\int_{e^{i d} \mathbb{R}_{+}} e_{m}(s / t) v(s) \frac{\mathrm{ds}}{s}
$$

is called an m-moment Laplace transform in a direction $d$.

- If $v \in \mathcal{O}\left(S_{d}\left(\frac{\pi}{k}+\varepsilon, R\right), \mathbb{E}\right)$ for some $\varepsilon, R>0$, then the integral operator $T_{m, d}^{-}$is defined as follows:

$$
\left(T_{m, d}^{-} v\right)(s):=-\frac{1}{2 \pi i} \int_{\gamma(d)} E_{m}(s / t) v(t) \frac{\mathrm{dt}}{t}
$$

(where a path $\gamma(d)$ is the boundary of a sector contained in $S_{d}\left(\frac{\pi}{k}+\varepsilon, R\right)$ with bisecting direction $d$, a finite radius, an opening slightly larger than $\pi / k$, and the orientation is negative) is called an inverse $m$-moment Laplace transform in a direction $d$.

Remark 5 Observe, that $T_{m, d}\left(t^{n}\right)=m(n) t^{n}$ for every $n \in \mathbb{N}_{0}$. Hence, $T_{m, d} \widehat{\mathcal{B}}_{m} u=u$ for every $u \in \mathcal{O}(D)$.

Now, we are ready to define the summability of formal power series.

Definition 8 Let $k>0$ and $d \in \mathbb{R}$. Then, $\widehat{u} \in \mathbb{E}[[t]]$ is called $k$-summable in a direction $d$ if there exist $\varepsilon>0$ and a disc-sector $\widehat{S}_{d}=\widehat{S}_{d}(\varepsilon)$ in a direction $d$ such that $v=\widehat{\mathcal{B}}_{\Gamma_{1 / k}} \widehat{u} \in$ $\mathcal{O}^{k}\left(\widehat{S}_{d}, \mathbb{E}\right)$.

Moreover, the $k$-sum of $\widehat{u}$ in the direction $d$ is given by the following:

$$
u^{d}(t)=\mathcal{S}_{k, d} \widehat{u}(t):=\left(T_{m, \theta} v\right)(t)=\int_{e^{i \theta} \mathbb{R}_{+}} e_{m}(s / t) v(s) \frac{\mathrm{ds}}{s} \quad \text { for } \quad \theta \in(d-\varepsilon / 2, d+\varepsilon / 2) .
$$

Definition 9 If $\widehat{u} \in \mathbb{E}[[t]]$ is $k$-summable in all directions $d$ but (after identification modulo $2 \pi$ ) finitely many directions $d_{1}, \ldots, d_{n}$, then $\widehat{u}$ is called $k$-summable and $d_{1}, \ldots, d_{n}$ are called singular directions of $\widehat{u}$.

Next, we extend the notion of $k$-summable formal power series to that which are multisummable. 
Definition 10 Let $k_{1}>\cdots>k_{n}>0$ and let $\kappa_{1}, \ldots, \kappa_{n}$ be defined by $\kappa_{1}=k_{1}, 1 / \kappa_{j}=$ $1 / k_{j}-1 / k_{j-1}, 2 \leq j \leq n$. We say that a real vector $\mathbf{d}=\left(d_{1}, \ldots, d_{n}\right)$ is an admissible multidirection with respect to $\mathbf{k}=\left(k_{1}, \ldots, k_{n}\right)$ if

$$
2 \kappa_{j}\left|d_{j}-d_{j-1}\right| \leq \pi \quad \text { for } \quad j=2, \ldots, n .
$$

Remark 6 Admissibility of $\mathbf{d}$ with respect to $\mathbf{k}$ is equivalent to the inclusions $I_{1} \subseteq I_{2} \subseteq$ $\cdots \subseteq I_{n}$, where $I_{j}:=\left(d_{j}-\frac{\pi}{2 k_{j}}, d_{j}+\frac{\pi}{2 k_{j}}\right)$ for $j=1, \ldots, n$.

Definition 11 Let $m_{1}, \ldots, m_{n}$ be moment functions of positive orders respectively $1 / \kappa_{1}, \ldots, 1 / \kappa_{n}$, where $\kappa_{1}, \ldots, \kappa_{n}$ are constructed in Definition 10. A formal power series $\widehat{u}(t)=\sum_{j=0}^{\infty} u_{j} t^{j} \in \mathbb{E}[[t]]$ is called $\boldsymbol{k}$-multisummable in the admissible multidirection, provided by the following:

- $\quad v_{n}(t):=\left(\widehat{\mathcal{B}}_{m_{n}} \cdots \widehat{\mathcal{B}}_{m_{1}} \widehat{u}\right)(t)=\sum_{j=0}^{\infty} \frac{u_{j}}{m_{1}(j) \cdots m_{n}(j)} t^{j} \in \mathcal{O}^{\kappa_{n}}\left(\widehat{S}_{d_{n}}\right)$.

- $v_{j-1}(t):=\left(T_{m_{j}, d_{j}} v_{j}\right)(t) \in \mathcal{O}^{\kappa_{j-1}}\left(S_{d_{j-1}}\right)$ for $j=n, n-1, \ldots, 2$.

Moreover, the k-multisum of $\widehat{u}$ in the multidirection $\mathrm{d}$ is given by the following:

$$
u^{\mathbf{d}}(t)=\mathcal{S}_{\mathbf{k}, \mathbf{d}} \widehat{u}(t):=\left(T_{m_{1}, d_{1}} \cdots T_{m_{n}, d_{n}} v_{n}\right)(t) .
$$

Definition 12 If $\left(d_{1}, \ldots, d_{n}\right)$ is an admissible multidirection and the functions $v_{n}, \ldots, v_{j}$ all exist, but $v_{j} \notin \mathcal{O}^{\kappa_{j}}\left(S_{d_{j}}\right)$, then $d_{j}$ is called a singular direction of $\widehat{u}$ of level $k_{j}$ (for $j=1, \ldots, n)$.

Definition 13 If $\widehat{u}$ has at most (after identification modulo $2 \pi$ ) finitely many singular directions of each level $k_{j}, 1 \leq j \leq n$, then $\widehat{u}$ is called $\boldsymbol{k}$-multisummable.

Remark 7 If $k_{1}>\cdots>k_{n}>0,\left(d_{1}, \ldots, d_{n}\right)$ is an admissible multidirection and $\widehat{u}_{j}$ is $k_{j}$ summable in a direction $d_{j}$ for $j=1, \ldots, n$, then, by [2, Lemma 20], $\widehat{u}:=\widehat{u}_{1}+\cdots+\widehat{u}_{n}$ is k-multisummable in the multidirection $\mathbf{d}$ and $\mathcal{S}_{\mathbf{k}, \mathbf{d}} \widehat{u}(t)=\mathcal{S}_{k_{1}, d_{1}} \widehat{u}_{1}(t)+\cdots+\mathcal{S}_{k_{n}, d_{n}} \widehat{u}_{n}(t)$.

Moreover, if additionally $\widehat{u}_{j}$ is $k_{j}$-summable with $n_{j}$ singular directions $d_{j, 1}, \ldots, d_{j, n_{j}}$ (for $j=1, \ldots, n$ ) then $\widehat{u}$ is $\mathbf{k}$-multisummable and $d_{j, 1}, \ldots, d_{j, n_{j}}$ are singular directions of $\widehat{u}$ of level $k_{j}$.

\section{Moment Operators}

In this section, we recall the notion of moment differential operators constructed by Balser and Yoshino [3] and the concept of moment pseudodifferential operators introduced in the previous papers of the first author $[15,16]$.

Definition 14 Let $m$ be a moment function. Then, the linear operator $\partial_{m, x}: \mathbb{E}[[x]] \rightarrow$ $\mathbb{E}[[x]]$ is defined by the following:

$$
\partial_{m, x}\left(\sum_{j=0}^{\infty} \frac{u_{j}}{m(j)} x^{j}\right):=\sum_{j=0}^{\infty} \frac{u_{j+1}}{m(j)} x^{j}
$$

and is called an m-moment differential operator $\partial_{m, x}$. 
Below, we present most important examples of moment differential operators. Other examples, including also integro-differential operators, can be found in [16, Example 3].

Example 3 If $m(u)=\Gamma_{1}(u)$, then the operator $\partial_{m, x}$ coincides with the usual differentiation $\partial_{x}$. More generally, if $s>0$ and $m(u)=\Gamma_{s}(u)$, then the operator $\partial_{m, x}$ satisfies $\left(\partial_{m, x} \widehat{u}\right)\left(x^{s}\right)=\partial_{x}^{s}\left(\widehat{u}\left(x^{s}\right)\right)$, where $\partial_{x}^{s}$ denotes the Caputo fractional derivative of order $s$ defined by the following:

$$
\partial_{x}^{s}\left(\sum_{j=0}^{\infty} \frac{u_{j}}{\Gamma_{s}(j)} x^{s j}\right):=\sum_{j=0}^{\infty} \frac{u_{j+1}}{\Gamma_{s}(j)} x^{s j} .
$$

Immediately by the definition, we obtain the following connection between the moment Borel transform and the moment differentiation.

Proposition 3 Let $m$ and $m^{\prime}$ be two moment functions. Then, the operators $\widehat{\mathcal{B}}_{m^{\prime}}, \partial_{m, t}: \mathbb{E}[[t]] \rightarrow \mathbb{E}[[t]]$ satisfy the following commutation formulas for every $\widehat{u} \in \mathbb{E}[[t]]$ and for $\bar{m}=\mathrm{mm}^{\prime}$ :

i) $\widehat{\mathcal{B}}_{m^{\prime}} \partial_{m, t} \widehat{u}=\partial_{\bar{m}, t} \widehat{\mathcal{B}}_{m^{\prime}} \widehat{u}$,

ii) $\widehat{\mathcal{B}}_{m^{\prime}} P\left(\partial_{m, t}\right) \widehat{u}=P\left(\partial_{\bar{m}, t}\right) \widehat{\mathcal{B}}_{m^{\prime}} \widehat{u}$ for any polynomial $P$ with constant coefficients.

Now, following [16], we generalise moment differential operators to a kind of pseudodifferential operators. Namely, we have the following:

Definition 15 ([16, Definition 13]) Let $m$ be a moment function of order $1 / k>0$ and $\lambda(\zeta)$ be an analytic function of the variable $\xi=\zeta^{1 / \gamma}$ for $|\zeta| \geq r_{0}$ (for some $\gamma \in \mathbb{N}$ and $r_{0}>0$ ) of polynomial growth at infinity. A moment pseudodifferential operator $\lambda\left(\partial_{m, z}\right): \mathcal{O}_{1 / \gamma}(D) \rightarrow$ $\mathcal{O}_{1 / \gamma}(D)$ is defined by the following:

$$
\lambda\left(\partial_{m, z}\right) \varphi(z):=\frac{1}{2 \gamma \pi i} \oint_{|w|=\varepsilon}^{\gamma} \varphi(w) \int_{r_{0} e^{i \theta}}^{\infty(\theta)} \lambda(\zeta) E_{\widetilde{m}}\left(\zeta^{1 / \gamma} z^{1 / \gamma}\right) \frac{e_{m}(\zeta w)}{\zeta w} d \zeta d w
$$

for every $\varphi \in \mathcal{O}_{1 / \gamma}\left(D_{r}\right)$ and $|z|<\varepsilon<r$, where $\tilde{m}(u):=m(u / \gamma), E_{\widetilde{m}}\left(\zeta^{1 / \gamma} z^{1 / \gamma}\right)=$ $\sum_{n=0}^{\infty} \frac{\zeta^{n / \gamma} z^{n / \gamma}}{m(n / \gamma)}, \theta \in\left(-\arg w-\frac{\pi}{2 k},-\arg w+\frac{\pi}{2 k}\right)$ and $\oint_{|w|=\varepsilon}^{\gamma}$ means that we integrate $\gamma$ times along the positively oriented circle of radius $\varepsilon$. Here, the integration in the inner integral is taken over a ray $\left\{r e^{i \theta}: r \geq r_{0}\right\}$.

Definition 16 [15, Definition 9] Let $\lambda(\zeta)$ be an analytic function of the variable $\xi=\zeta^{1 / \gamma}$ for $|\zeta| \geq r_{0}$ (for some $\gamma \in \mathbb{N}$ and $r_{0}>0$ ) of polynomial growth at infinity. Then, we define the pole order $q \in \mathbb{Q}$ and the leading term $\lambda_{0} \in \mathbb{C} \backslash\{0\}$ of $\lambda(\zeta)$ as the numbers satisfying the formula $\lim _{\zeta \rightarrow \infty} \lambda(\zeta) / \zeta^{q}=\lambda_{0}$. We write it also $\lambda(\zeta) \sim \lambda_{0} \zeta^{q}$.

\section{Stokes Phenomenon and Hyperfunctions}

Now, we extend the concept of the Stokes phenomenon (see [18, Definition 7]) to multisummable formal power series $\widehat{u} \in \mathbb{E}[[t]]$. 
Definition 17 Assume that $\widehat{u} \in \mathbb{E}[[t]]$ is k-multisummable with singular directions $d_{j, 1}, \ldots, d_{j, n_{j}}$ of level $k_{j}, 1 \leq j \leq n$. Then, for every $l=1, \ldots, n_{j}$ and $j=1, \ldots, n$, the set $\mathcal{L}_{d_{j, l}}=\left\{t \in \tilde{\mathbb{C}}: \arg t=d_{j, l}\right\}$ is called a Stokes line of level $k_{j}$ for $\widehat{u}$.

Assume now that for fixed $j \in\{1, \ldots, n\}$ the vector $\mathbf{d}=\left(d_{1}, \ldots, d_{n}\right)$ is an admissible multidirection with a singular direction $d_{j}$ of level $k_{j}$ and with nonsingular directions $d_{l}$ of level $k_{l}$ for $l \neq j$, and let $\mathbf{d}_{\mathbf{j}}^{ \pm}:=\left(d_{1}, \ldots, d_{j}^{ \pm}, \ldots, d_{n}\right)$ be the admissible multidirections, where $d_{j}^{+}$(resp. $d_{j}^{-}$) denotes a direction close to $d_{j}$ and greater (resp. less) than $d_{j}$, and let $u^{\mathbf{d}_{\mathbf{j}}^{+}}:=\mathcal{S}_{\mathbf{k}, \mathbf{d}_{\mathbf{j}}^{+}} \widehat{u}\left(\right.$ resp. $\left.u^{\mathbf{d}_{\mathbf{j}}^{-}}:=\mathcal{S}_{\mathbf{k}, \mathbf{d}_{\mathbf{j}}^{-}} \widehat{u}\right)$ then the difference $J_{\mathcal{L}_{d_{j}}, k_{j}} \widehat{u}:=u^{\mathbf{d}_{\mathbf{j}}^{+}}-u^{\mathbf{d}_{\mathbf{j}}^{-}}$is called a jump for $\widehat{u}$ across the Stokes line $\mathcal{L}_{d_{j}}$ of level $k_{j}$.

Remark 8 Every Stokes line $\mathcal{L}_{d_{j}}$ of level $k_{j}$ for $\widehat{u}$ determines also so called anti-Stokes lines $\mathcal{L}_{d_{j} \pm \frac{\pi}{2 k_{j}}}$ of level $k_{j}$ for $\widehat{u}$.

We will describe jumps across the Stokes lines in terms of hyperfunctions. The similar approach to the Stokes phenomenon one can find in $[8,13,20]$. For more information about the theory of hyperfunctions, we refer the reader to [9].

We will consider the space as follows:

$$
\mathcal{H}^{k}\left(\mathcal{L}_{d}\right):=\mathcal{O}^{k}\left(D \cup\left(S_{d} \backslash \mathcal{L}_{d}\right)\right) / \mathcal{O}^{k}\left(\widehat{S}_{d}\right)
$$

of Laplace-type hyperfunctions supported by $\mathcal{L}_{d}$ with exponential growth of order $k$. It means that every hyperfunction $G \in \mathcal{H}^{k}\left(\mathcal{L}_{d}\right)$ may be written as follows:

$$
G(s)=[g(s)]_{d}=\left\{g(s)+h(s): h(s) \in \mathcal{O}^{k}\left(\widehat{S}_{d}\right)\right\}
$$

for some defining function $g(s) \in \mathcal{O}^{k}\left(D \cup\left(S_{d} \backslash \mathcal{L}_{d}\right)\right)$.

Let $\gamma_{d}$ be a path consisting of the half-lines from $e^{i d^{-}} \infty$ to 0 and from 0 to $e^{i d^{+}} \infty$, i.e. $\gamma_{d}=-\gamma_{d^{-}}+\gamma_{d^{+}}$with $\gamma_{d^{ \pm}}=\mathcal{L}_{d^{ \pm}}$. By the Köthe-type theorem [10], one can treat the hyperfunction $G(s)=[g(s)]_{d}$ as the analytic functional defined by the following:

$$
G(s)[\varphi(s)]:=\int_{\gamma_{d}} g(s) \varphi(s) \mathrm{ds},
$$

for such small $\varphi \in \mathcal{O}^{-k}\left(\widehat{S}_{d}\right)$ that the function $s \mapsto g(s) \varphi(s)$ belongs to the space $\mathcal{O}^{-k}(D \cup$ $\left.\left(S_{d} \backslash \mathcal{L}_{d}\right)\right)$.

To describe the jumps across the Stokes lines in terms of hyperfunctions, first assume that $\widehat{f} \in \mathbb{C}[[t]]$ is $k$-summable, $m$ is a moment function of order $1 / k$ and $d$ is a singular direction. By Eq. 4, the jump for $\widehat{f}$ across the Stokes line $\mathcal{L}_{d}$ is given by the following:

$$
J_{\mathcal{L}_{d}} \widehat{f}(t)=f^{d^{+}}(t)-f^{d^{-}}(t)=\left(T_{m, d^{+}}-T_{m, d^{-}}\right) \widehat{\mathcal{B}}_{m} \widehat{f}(t) .
$$

Observe that we can treat $g_{0}(t):=\widehat{\mathcal{B}}_{m} \widehat{f}(t) \in \mathcal{O}^{k}\left(D \cup\left(S_{d} \backslash \mathcal{L}_{d}\right)\right)$ as a defining function of the hyperfunction $G_{0}(s):=\left[g_{0}(s)\right]_{d} \in \mathcal{H}^{k}\left(\mathcal{L}_{d}\right)$. So, combining Eq. 5 with Eq. 6, we conclude that

$$
J_{\mathcal{L}_{d}} \widehat{f}(t)=G_{0}(s)\left[\frac{e_{m}(s / t)}{s}\right] \text { for sufficiently small } r>0 \text { and } t \in S_{d}\left(\frac{\pi}{k}, r\right) .
$$

Moreover, it is natural to define the $m$-moment Laplace operator $T_{m, d}$ acting on the hyperfunction $G(s)$ as $T_{m, d} G(t):=G(s)\left[\frac{e_{m}(s / t)}{s}\right]$ for $t \in S_{d}\left(\frac{\pi}{k}, r\right)$, where $G(s)[\varphi(s)]$ is defined by Eq. 5. So, by Eq. 7, we may describe the jump in terms of the $m$-moment Laplace operator acting on the hyperfunction as $J_{\mathcal{L}_{d}} \widehat{f}(t)=T_{m, d} G_{0}(t)$. 
Now, let $\widehat{f} \in \mathbb{C}[[t]]$ be k-multisummable and $\mathbf{d}$ be as in Definition 17 with $\mathcal{L}_{d_{j}}$ being the Stokes line of level $k_{j}$. We additionally assume as in Remark 7 that $\widehat{f}=\widehat{f_{1}}+\cdots+\widehat{f}_{n}$, where $\widehat{f_{j}}$ is $k_{j}$-summable. Then, by Remark 7, analogously as in the summable case, the jump across $\mathcal{L}_{d_{j}}$ of level $k_{j}$ is given by the following:

$$
J_{\mathcal{L}_{d_{j}}, k_{j}} \widehat{f}=f^{\mathbf{d}_{\mathbf{j}}^{+}}-f^{\mathbf{d}_{\mathbf{j}}^{-}}=f_{j}^{d_{j}^{+}}-f_{j}^{d_{j}^{-}}=\left(T_{m_{j}, d_{j}^{+}}-T_{m_{j}, d_{j}^{-}}\right) \widehat{\mathcal{B}}_{m_{j}} \widehat{f}_{j},
$$

and we may describe this jump in terms of hyperfunctions as in the previous case.

Similarly, if $\mathcal{L}_{d}$ is a Stokes line for $k$-summable $\widehat{u}=\widehat{u}(t, z) \in \mathcal{O}(D)[[t]]$, then we are able to describe jumps for $\widehat{u}(t, z)$ at the point $z=0$ in terms of hyperfunctions. Namely, we have the following:

$$
\begin{aligned}
J_{\mathcal{L}_{d}} \widehat{u}(t, 0) & =\left(T_{m, d} F_{0}\right)(t)=F_{0}(s)\left[\frac{e_{m}(s / t)}{s}\right], \quad \text { where } F_{0}(s) \\
& =\left[\widehat{\mathcal{B}}_{m} \widehat{u}(s, 0)\right]_{d} \in \mathcal{H}^{k}\left(\mathcal{L}_{d}\right) .
\end{aligned}
$$

Analogously, we calculate jumps across a Stokes line $\mathcal{L}_{d_{j}}$ of level $k_{j}$ for $\mathbf{k}$ multisummable $\widehat{u}$ satisfying $\widehat{u}=\widehat{u}_{1}+\cdots+\widehat{u}_{n}$, where $\widehat{u}_{i}$ is $k_{i}$-summable $(i=1, \ldots, n)$.

Remark 9 In some special cases, we are also able to describe jumps for $\widehat{u}(t, z)$ at any point $z \in D$. It is possible in the case when $\widehat{u}$ is a multisummable solution of

$$
\left\{\begin{array}{l}
P\left(\partial_{m_{1}, t}, \partial_{z}\right) u=0 \\
\partial_{m_{1}, t}^{j} u(0, z)=\varphi_{j}(z) \quad \text { for } \quad j=0, \ldots, N-1,
\end{array}\right.
$$

instead of Eq. 1. In this case, we are able to reduce the problem of description of jumps for $\widehat{u}(t, z)$ at the fixed point $z \in D$, to the problem of description of jumps for the auxiliary formal power series $\widehat{u}_{z}(t, s):=\widehat{u}(t, s+z)$ at the point $s=0$. Since the derivative operator $\partial_{z}$ is invariant under the translation, i.e. $\left(\partial_{z} \widehat{u}\right)(t, s+z)=\partial_{s}(\widehat{u}(t, s+z))$, we conclude that $\widehat{u}_{z}(t, s)$ is a multisummable solution of

$$
\left\{\begin{array}{l}
P\left(\partial_{m_{1}, t}, \partial_{s}\right) u_{z}=0 \\
\partial_{m_{1}, t}^{j} u_{z}(0, s)=\varphi_{z, j}(s):=\varphi_{j}(s+z) \text { for } \quad j=0, \ldots, N-1 .
\end{array}\right.
$$

Hence, $J_{\mathcal{L}_{d}} \widehat{u}(t, z)=J_{\mathcal{L}_{d z}} \widehat{u}_{z}(t, 0)$, where $\mathcal{L}_{d_{z}}$ is a Stokes line of $\widehat{u}_{z}$, which corresponds to a Stokes line $\mathcal{L}_{d}$ of $\widehat{u}$.

Since in general the moment differential operators are not invariant under translation, we are not able to use this method to describe the jumps for solutions of $P\left(\partial_{m_{1}, t}, \partial_{m_{2}, z}\right) u=0$ at any point $z \in D$.

\section{A Maximal Family of Solutions}

Now, we are ready to describe a family of normalised actual solutions of given nonKowalevskian equation using sums of multisummable formal power series solution. More precisely, we consider the Cauchy problem

$$
\left\{\begin{array}{l}
P\left(\partial_{m_{1}, t}, \partial_{m_{2}, z}\right) u=0 \\
\partial_{m_{1}, t}^{j} u(0, z)=\varphi_{j}(z) \in \mathcal{O}(D), \quad j=0, \ldots, N-1,
\end{array}\right.
$$


where $m_{1}, m_{2}$ are moment functions of orders $s_{1}, s_{2}>0$ respectively and

$$
P(\lambda, \zeta)=P_{0}(\zeta) \lambda^{N}-\sum_{j=1}^{N} P_{j}(\zeta) \lambda^{N-j}
$$

is a general polynomial of two variables, which is of order $N$ with respect to $\lambda$.

If $P_{0}(\zeta)$ defined by Eq. 9 is not a constant, then a formal solution of Eq. 8 is not uniquely determined. To avoid this inconvenience, we choose some special solution which is already uniquely determined. To this end, we factorise the polynomial $P(\lambda, \zeta)$ as follows:

$$
P(\lambda, \zeta)=P_{0}(\zeta)\left(\lambda-\lambda_{1}(\zeta)\right)^{N_{1}} \cdots\left(\lambda-\lambda_{l}(\zeta)\right)^{N_{l}}=: P_{0}(\zeta) \widetilde{P}(\lambda, \zeta),
$$

where $\lambda_{1}(\zeta), \ldots, \lambda_{l}(\zeta)$ are the roots of the characteristic equation $P(\lambda, \zeta)=0$ with multiplicity $N_{1}, \ldots, N_{l}\left(N_{1}+\cdots+N_{l}=N\right)$ respectively.

Since $\lambda_{\alpha}(\zeta)$ are algebraic functions, we may assume that there exist $\gamma \in \mathbb{N}$ and $r_{0}<\infty$ such that $\lambda_{\alpha}(\zeta)$ are holomorphic functions of the variable $\xi=\zeta^{1 / \gamma}$ (for $|\zeta| \geq r_{0}$ and $\alpha=1, \ldots, l$ ) and, moreover, there exist $\lambda_{\alpha} \in \mathbb{C} \backslash\{0\}$ and $q_{\alpha}=\mu_{\alpha} / v_{\alpha}$ (for some relatively prime numbers $\mu_{\alpha} \in \mathbb{Z}$ and $v_{\alpha} \in \mathbb{N}$ ) such that $\lambda_{\alpha}(\zeta) \sim \lambda_{\alpha} \zeta^{q_{\alpha}}$ for $\alpha=1, \ldots, l$. Observe that $v_{\alpha} \mid \gamma$ for $\alpha=1, \ldots, l$.

Hence, $\lambda_{\alpha}\left(\partial_{m_{2}, z}\right)$ are well-defined moment pseudodifferential operators and consequently also the operator

$$
\widetilde{P}\left(\partial_{m_{1}, t}, \partial_{m_{2}, z}\right)=\left(\partial_{m_{1}, t}-\lambda_{1}\left(\partial_{m_{2}, z}\right)\right)^{N_{1}} \cdots\left(\partial_{m_{1}, t}-\lambda_{l}\left(\partial_{m_{2}, z}\right)\right)^{N_{l}}
$$

is well-defined.

Under the above assumption, by a normalised formal solution $\widehat{u}$ of Eq. 8, we mean such solution of Eq. 8, which is also a solution of the pseudodifferential equation $\widetilde{P}\left(\partial_{m_{1}, t}, \partial_{m_{2}, z}\right) \widehat{u}=0$ (see [15, Definition 10]).

Since the principal part of the pseudodifferential operator $\widetilde{P}\left(\partial_{m_{1}, t}, \partial_{m_{2}, z}\right)$ with respect to $\partial_{m_{1}, t}$ is given by $\partial_{m_{1}, t}^{N}$, the Cauchy problem (8) has a unique normalised formal power series solution $\widehat{u} \in \mathcal{O}(D)[[t]]$. If we additionally assume that $\widehat{u}$ is multisummable, then using the procedure of multisummability in nonsingular directions, we obtain a family of normalised actual solutions of Eq. 8 on some sectors with respect to $t$. This motivates us to introduce the following definitions.

Definition 18 Let $S$ be a sector in the universal covering space $\tilde{\mathbb{C}}$. A function $u \in \mathcal{O}(S \times D)$ is called a normalised actual solution of Eq. 8 if it satisfies

$$
\left\{\begin{array}{l}
\widetilde{P}\left(\partial_{m_{1}, t}, \partial_{m_{2}, z}\right) u=0 \\
\lim _{t \rightarrow 0, t \in S} \partial_{m_{1}, t}^{j} u(t, z)=\varphi_{j}(z) \in \mathcal{O}(D), \quad j=0, \ldots, N-1 .
\end{array}\right.
$$

In [18], we introduced a maximal family of solutions of Eq. 8 in the case when a formal power series solution is $k$-summable. It is a collection of all actual solutions of Eq. 8 constructed by the procedure of $k$-summability. Now, we generalise this definition to the multisummable case.

Definition 19 Assume that the normalised formal power series solution $\widehat{u}$ of Eq. 8 is $\mathbf{k}$ multisummable, $\mathcal{J}$ is a finite set of indices, and $V$ is a sector with an opening greater than $\pi / k_{n}$ on the Riemann surface of $t^{\frac{1}{q}}$ for some $q \in \mathbb{Q}_{+}$.

We say that $\left\{u_{i}\right\}_{i \in \mathcal{J}}$ with $u_{i} \in \mathcal{O}\left(V_{i} \times D\right)$ is a maximal family of solutions of Eq. 8 on $V \times D$ if the following conditions hold: 
(a) $\quad V_{i} \subseteq V$ is a sector of opening greater than $\pi / k_{1}$ for every $i \in \mathcal{J}$.

(b) $\left\{V_{i}\right\}_{i \in \mathcal{J}}$ is a covering of $V$.

(c) $u_{i} \in \mathcal{O}\left(V_{i} \times D\right)$ is a normalised actual solution of Eq. 8 for every $i \in \mathcal{J}$.

(d) If $V_{i} \cap V_{j} \neq \emptyset$ then $u_{i} \not \equiv u_{j}$ on $\left(V_{i} \cap V_{j}\right) \times D$ for every $i, j \in \mathcal{J}, i \neq j$.

(e) For every $i \in \mathcal{J}$, there exists an admissible nonsingular multidirection $\mathbf{d}$ such that $u_{i}=\mathcal{S}_{\mathbf{k}, \mathbf{d}} \widehat{u}$ on $\tilde{V} \times D$ for some non-empty sector $\tilde{V} \subseteq V_{i}$.

(f) For every admissible nonsingular multidirection d, there exists $i \in \mathcal{J}$ such that $\mathcal{S}_{\mathbf{k}, \mathbf{d}} \widehat{u}=u_{i}$ on $\tilde{V} \times D$ for some sector $\tilde{V} \subseteq V_{i}$.

Now, we are ready to describe a maximal family of solutions of Eq. 8 generalising our previous result [18, Theorem 3$]$ to the multisummable case.

Theorem 1 Let $\widehat{u}$ be a $\mathbf{k}$-multisummable normalised formal power series solution of Eq. 8 with a $\mathbf{k}$-multisum in a nonsingular admissible multidirection $\mathbf{d}$ given by $u^{\mathbf{d}}=\mathcal{S}_{\mathbf{k}, \mathbf{d}} \widehat{u}$ and satisfying $\widehat{u}=\widehat{u}_{1}+\cdots+\widehat{u}_{n}$, where $\widehat{u}_{j}$ is $k_{j}$-summable for $j=1, \ldots, n$. Assume that there exists $q \in \mathbb{Q}_{+}$, which is the smallest positive rational number such that $u^{\mathbf{d}}(t, z)=$ $u^{\mathbf{d}}\left(t e^{2 q \pi i}, z\right)$ for every nonsingular multidirection $\mathbf{d}$. Suppose that the set of singular directions of $\widehat{u}$ of level $k_{j}$ modulo $2 q \pi$ is given by $\left\{d_{j, 1}, \ldots, d_{j, n_{j}}\right\}$, where $0 \leq d_{j, 1}<\cdots<$ $d_{j, n_{j}}<2 q \pi(j=1, \ldots, n)$.

Furthermore, let

$$
I_{j, l}:=\left(d_{j, l}-\frac{\pi}{2 k_{j}}, d_{j, l+1}+\frac{\pi}{2 k_{j}}\right) \quad \text { for } \quad l=1, \ldots, n_{j}, \quad j=1, \ldots, n,
$$

where $d_{j, n_{j}+1}:=d_{j, 1}+2 q \pi$, and let

$$
\mathcal{J}:=\left\{\mathbf{l}=\left(l_{1}, \ldots, l_{n}\right) \in \mathbb{N}^{n}: 1 \leq l_{j} \leq n_{j},\left|I_{j, l_{j}} \cap \ldots \cap I_{n, l_{n}}\right|>\frac{\pi}{k_{j}}, j=1, \ldots, n\right\},
$$

where $|I|$ denotes the length of the interval I.

Then,

(i) for every $\mathbf{l} \in \mathcal{J}$ there exists an admissible multidirection $\mathbf{d}=\left(d_{1}, \ldots, d_{n}\right)$ satisfying

$$
d_{j} \in\left(d_{j, l_{j}}, d_{j, l_{j}+1}\right), \quad j=1, \ldots, n,
$$

for which the function $u_{\mathbf{l}}:=\mathcal{S}_{\mathbf{k}, \mathbf{d}} \widehat{u}$ is well-defined.

(ii) For every sufficiently small $\varepsilon>0$, there exists $r>0$ such that $u_{\mathbf{l}} \in \mathcal{O}\left(V_{\mathbf{l}}(\varepsilon, r) \times D\right)$ for every $\mathbf{l} \in \mathcal{J}$, where

$$
V_{\mathbf{l}}(\varepsilon, r):=\left\{t \in W_{r}:\left(\arg t-\frac{\varepsilon}{2}, \arg t+\frac{\varepsilon}{2}\right) \subseteq I_{1, l_{1}} \cap \cdots \cap I_{n, l_{n}}\right\}
$$

and $W_{r}=\{t \in W: 0<|t|<r\}$ with $W$ being the Riemann surface of $t \mapsto t^{\frac{1}{q}}$.

(iii) $\left\{u_{\mathbf{l}}\right\}_{\mathbf{l} \in \mathcal{J}}$ is a maximal family of solutions of Eq. 8 on $W_{r} \times D$.

Remark 10 Observe that $\mathcal{L}_{d_{j, l}}$ and $\mathcal{L}_{d_{j, l} \pm \frac{\pi}{2 k_{j}}}$, with $d_{j, l}$ satisfying the assumptions of Theorem 1 , are respectively Stokes and anti-Stokes lines of level $j$ for $l=1, \ldots, n_{j}$ and $j=1, \ldots, n$. They play an important role in our description of the maximal family of solutions of Eq. 8 . 
Proof of Theorem 1 (i) First, observe that condition

$$
\left|I_{j, l_{j}} \cap \cdots \cap I_{n, l_{n}}\right|>\frac{\pi}{k_{j}} \quad \text { for } \quad j=1, \ldots, n
$$

guarantees that there exist $d_{j} \in\left(d_{j, l_{j}}, d_{j, l_{j}+1}\right), j=1, \ldots, n$, such that

$$
\left(d_{1}-\frac{\pi}{2 k_{1}}, d_{1}+\frac{\pi}{2 k_{1}}\right) \subseteq\left(d_{2}-\frac{\pi}{2 k_{2}}, d_{2}+\frac{\pi}{2 k_{2}}\right) \subseteq \cdots \subseteq\left(d_{n}-\frac{\pi}{2 k_{n}}, d_{n}+\frac{\pi}{2 k_{n}}\right) .
$$

It means that for every $\mathbf{l} \in \mathcal{J}$, one can find an admissible multidirection $\mathbf{d}=\left(d_{1}, \ldots, d_{n}\right)$ satisfying (10).

Moreover, for every admissible multidirections $\mathbf{d}=\left(d_{1}, \ldots, d_{n}\right)$ and $\tilde{\mathbf{d}}=\left(\tilde{d}_{1}, \ldots, \tilde{d}_{n}\right)$ such that $d_{j}, \tilde{d}_{j} \in\left(d_{j, l_{j}}, d_{j, l_{j}+1}\right)$ for $j=1, \ldots, n$ we have as follows:

$$
\mathcal{S}_{\mathbf{k}, \mathbf{d}} \widehat{u}=\mathcal{S}_{k_{1}, d_{1}} \widehat{u}_{1}+\cdots+\mathcal{S}_{k_{n}, d_{n}} \widehat{u}_{n}=\mathcal{S}_{k_{1}, \tilde{d}_{1}} \widehat{u}_{1}+\cdots+\mathcal{S}_{k_{n}, \tilde{d}_{n}} \widehat{u}_{n}=\mathcal{S}_{\mathbf{k}, \tilde{\mathbf{d}}} \widehat{u},
$$

since by [2, Lemma 10]

$$
\mathcal{S}_{k_{j}, d_{j}} \widehat{u}_{j}=\mathcal{S}_{k_{j}, \tilde{d}_{j}} \widehat{u}_{j} \quad \text { for every } \quad j=1, \ldots, n .
$$

It means that for every $\mathbf{l} \in \mathcal{J}$, the function $u_{\mathbf{l}}$ is well-defined.

To show (ii), observe that for every sufficiently small $\varepsilon>0$ there exists $r>0$ such that $\mathcal{S}_{k_{j}, d_{j}} \widehat{u}_{j}$ is analytically continued to the set as follows:

$$
\left\{t \in W:|t| \in(0, r),\left(\arg t-\frac{\varepsilon}{2}, \arg t+\frac{\varepsilon}{2}\right) \subseteq I_{j, l_{j}}\right\} \times D \quad \text { for every } \quad j=1, \ldots, n .
$$

Hence, the whole function $u_{\mathbf{l}}$ is analytically continued to the set $V_{\mathbf{l}}(\varepsilon, r) \times D$.

Finally, we prove (iii). Since the inequality $\left|I_{1, l_{1}} \cap \cdots \cap I_{n, l_{n}}\right|>\frac{\pi}{k_{1}}$ holds for every $\mathbf{l} \in \mathcal{J}$, we are able to take such small $\varepsilon>0$ that the opening of $V_{\mathbf{l}}(\varepsilon, r)$ ( $V_{\mathbf{l}}$ for short) is greater than $\frac{\pi}{k_{1}}$.

We claim that $\left\{V_{\mathbf{l}}\right\}_{\mathbf{l} \in \mathcal{J}}$ is a covering of $W_{r}$. To this end, we take any $t \in W_{r}$. Then, we may choose $\mathbf{l} \in \mathbb{N}^{n}$ such that $1 \leq l_{j} \leq n_{j}$ and $\arg t \in\left[d_{j, l_{j}}, d_{j, l_{j}+1}\right)$ for $j=1, \ldots, n$. For such choice of $\mathbf{I}$ there exists $\delta>0$ such that

$$
\left[\arg t-\frac{\pi}{2 k_{j}}+\frac{\delta}{2}, \arg t+\frac{\pi}{2 k_{j}}+\delta\right] \subset I_{j, l_{j}} \cap \cdots \cap I_{n, l_{n}} \quad \text { for } \quad j=1, \ldots, n .
$$

It means that $\left|I_{j, l_{j}} \cap \cdots \cap I_{n, l_{n}}\right| \geq \frac{\pi}{k_{j}}+\frac{\delta}{2}>\frac{\pi}{k_{j}}$ for $j=1, \ldots, n$, so $\mathbf{l} \in \mathcal{J}$ and $t \in V_{\mathbf{l}}$. By the freedom of choice of $t \in W_{r},\left\{V_{\mathbf{l}}\right\}_{\mathbf{l} \in \mathcal{J}}$ is a covering of $W_{r}$.

By the moment version of [1, Theorem 6.2], we conclude that the space of $\mathbf{k}$ multisummable series in a multidirection $\mathbf{d}$ is a moment differential algebra over $\mathbb{C}$. It means that it is a linear space, which is also closed under multiplication and moment differentiations, and which for any k-multisummable series $\widehat{f}$ and $\widehat{g}$ satisfies: $\mathcal{S}_{\mathbf{k}, \mathbf{d}}(\widehat{f}+$ $\widehat{g})=\mathcal{S}_{\mathbf{k}, \mathbf{d}} \widehat{f}+\mathcal{S}_{\mathbf{k}, \mathbf{d}} \widehat{g}, \mathcal{S}_{\mathbf{k}, \mathbf{d}}(\widehat{f} \cdot \widehat{g})=\mathcal{S}_{\mathbf{k}, \mathbf{d}} \widehat{f} \cdot \mathcal{S}_{\mathbf{k}, \mathbf{d}} \widehat{g}, \mathcal{S}_{\mathbf{k}, \mathbf{d}}\left(\partial_{m_{1}, t} \widehat{f}\right)=\partial_{m_{1}, t}\left(\mathcal{S}_{\mathbf{k}, \mathbf{d}} \widehat{f}\right)$, and $\mathcal{S}_{\mathbf{k}, \mathbf{d}}\left(\partial_{m_{2}, z} \widehat{f}\right)=\partial_{m_{2}, z}\left(\mathcal{S}_{\mathbf{k}, \mathbf{d}} \widehat{f}\right)$.

Hence,

$$
P\left(\partial_{m_{1}, t}, \partial_{m_{2}, z}\right) u_{\mathbf{l}}=P\left(\partial_{m_{1}, t}, \partial_{m_{2}, z}\right) \mathcal{S}_{\mathbf{k}, \mathbf{d}} \widehat{u}=\mathcal{S}_{\mathbf{k}, \mathbf{d}} P\left(\partial_{m_{1}, t}, \partial_{m_{2}, z}\right) \widehat{u}=0 \text { on } V_{\mathbf{l}} \times D .
$$

Additionally, since $\widehat{u}(t, z)=\sum_{j=0}^{\infty} u_{j}(z) t^{j}$ on $V_{\mathbf{l}} \times D$, by [2, Proposition 8] and by the definition of multisummable series we get the following:

$$
\lim _{t \rightarrow 0, t \in V_{\mathbf{l}}} \partial_{m_{1}, t}^{j} u_{\mathbf{l}}(t, z)=\frac{m_{1}(j)}{m_{1}(0)} u_{j}(z)=\varphi_{j}(z) \quad \text { for } \quad j=0, \ldots, N-1 .
$$

Therefore $u_{\mathbf{l}}$ is an actual solution of Eq. 8 for $\mathbf{l} \in \mathcal{J}$. 
Now, assume that $V_{\mathbf{l}} \cap V_{\tilde{\mathbf{l}}} \neq \varnothing$ and $u_{\mathbf{l}} \equiv u_{\tilde{\mathbf{l}}}$ on $\left(V_{\mathbf{l}} \cap V_{\tilde{\mathbf{l}}}\right) \times D$ for some $\mathbf{l}, \tilde{\mathbf{l}} \in \mathcal{J}$ and $\mathbf{l} \neq \tilde{\mathbf{l}}$. It means that there exists admissible multidirections $\mathbf{d}=\left(d_{1}, \ldots, d_{n}\right), d_{j} \in\left(d_{j, l_{j}}, d_{j, l_{j+1}}\right)$ and $\tilde{\mathbf{d}}=\left(\tilde{d}_{1}, \ldots, \tilde{d}_{n}\right), \tilde{d}_{j} \in\left(d_{j, \tilde{l}_{j}}, d_{j, \tilde{l}_{j+1}}\right)$ such that

$$
u_{\mathbf{1}}=\mathcal{S}_{k_{1}, d_{1}} \widehat{u}_{1}+\cdots+\mathcal{S}_{k_{n}, d_{n}} \widehat{u}_{n}=\mathcal{S}_{k_{1}, \tilde{d}_{1}} \widehat{u}_{1}+\cdots+\mathcal{S}_{k_{n}, \tilde{d}_{n}} \widehat{u}_{n}=u_{\tilde{\mathbf{I}}} \text {. }
$$

Since $\mathcal{S}_{k_{j}, d_{j}} \widehat{u}_{j}$ and $\mathcal{S}_{k_{j}, \tilde{d}_{j}} \widehat{u}_{j}$ are both analytic on the non-empty

$$
\left\{t \in W:|t| \in(0, r),\left(\arg t-\frac{\varepsilon}{2}, \arg t+\frac{\varepsilon}{2}\right) \subseteq I_{j, l_{j}} \cap I_{j, \tilde{l}_{j}}\right\} \times D,
$$

by the Relative Watson's lemma [14, Proposition 2.1], we conclude that $\mathcal{S}_{k_{j}, d_{j}} \widehat{u}_{j}=$ $\mathcal{S}_{k_{j}, \tilde{d}_{j}} \widehat{u}_{j}$ for $j=1, \ldots, n$. Since $\mathbf{l} \neq \tilde{\mathbf{l}}$, without loss of generality, we may assume that $\tilde{d}_{i}<d_{i, l_{i}}<d_{i}$ for some $i \in\{1, \ldots, n\}$. This contradicts the fact that $d_{i, l_{i}}$ is a singular direction of level $k_{i}$. So if $V_{\mathbf{l}} \cap V_{\tilde{\mathbf{l}}} \neq \emptyset$ then $u_{\mathbf{l}} \neq u_{\tilde{\mathbf{l}}}$ on $\left(V_{\mathbf{l}} \cap V_{\tilde{\mathbf{l}}}\right) \times D$ for every $\mathbf{l}, \tilde{\mathbf{l}} \in \mathcal{J}, \mathbf{l} \neq \tilde{\mathbf{l}}$.

By the construction of the family $\left\{u_{\mathbf{l}}\right\}_{\mathbf{l} \in \mathcal{J}}$, the last two conditions in Definition 19 are also satisfied, which completes the proof.

\section{General Linear Moment Partial Differential Equations with Constant Coefficients}

We will study the Stokes phenomenon and the maximal family of solutions for the normalised formal solution $\widehat{u}$ of Eq. 8. Let us recall that we may reduce the Cauchy problem (8) of a general linear moment partial differential equation with constant coefficients to a family of the Cauchy problems of simple moment pseudodifferential equations. Namely, we have

Proposition 4 [16, Theorem 1] Let $\widehat{u}$ be the normalised formal solution of Eq. 8. Then, $\widehat{u}=\sum_{\alpha=1}^{l} \sum_{\beta=1}^{N_{\alpha}} \widehat{u}_{\alpha \beta}$ with $\widehat{u}_{\alpha \beta}$ being a formal solution of a simple pseudodifferential equation

$$
\left\{\begin{array}{l}
\left(\partial_{m_{1}, t}-\lambda_{\alpha}\left(\partial_{m_{2}, z}\right)\right)^{\beta} u_{\alpha \beta}=0 \\
\partial_{m_{1}, t}^{j} u_{\alpha \beta}(0, z)=0(j=0, \ldots, \beta-2) \\
\partial_{m_{1}, t}^{\beta-1} \widehat{u}_{\alpha \beta}(0, z)=\lambda_{\alpha}^{\beta-1}\left(\partial_{m_{2}, z}\right) \varphi_{\alpha \beta}(z),
\end{array}\right.
$$

where $\varphi_{\alpha \beta}(z):=\sum_{j=0}^{N-1} d_{\alpha \beta j}\left(\partial_{m_{2}, z}\right) \varphi_{j}(z) \in \mathcal{O}_{1 / \gamma}(D)$ and $d_{\alpha \beta j}(\zeta)$ are some holomorphic functions of the variable $\xi=\zeta^{1 / \gamma}$ and of polynomial growth.

Moreover, if $q_{\alpha}$ is a pole order of $\lambda_{\alpha}(\zeta)$ and $\bar{q}_{\alpha}=\max \left\{0, q_{\alpha}\right\}$, then $\widehat{u}_{\alpha \beta} \in$ $\mathcal{O}_{1 / \gamma}(D)[[t]]_{\bar{q}_{\alpha} s_{2}-s_{1}}$.

For this reason, we will study the following simple moment pseudodifferential equation

$$
\left\{\begin{array}{l}
\left(\partial_{m_{1}, t}-\lambda\left(\partial_{m_{2}, z}\right)\right)^{\beta} u=0 \\
\partial_{m_{1}, t}^{j} u(0, z)=0(j=0, \ldots, \beta-2) \\
\partial_{m_{1}, t}^{\beta-1} u(0, z)=\lambda^{\beta-1}\left(\partial_{m_{2}, z}\right) \varphi(z) \in \mathcal{O}_{1 / \gamma}(D),
\end{array}\right.
$$

where $m_{1}, m_{2}$ are moment functions of orders respectively $s_{1}, s_{2}>0$ such that $q s_{2}>s_{1}$, $\gamma \in \mathbb{N}, \lambda(\zeta) \sim \lambda_{0} \zeta^{q}$ with $q=\mu / v$ for some relatively prime $\mu, v \in \mathbb{N}$ satisfying $q \gamma \in \mathbb{N}$.

We start from the following representation of summable solutions of Eq. 11. 
Proposition 5 Let $d \in \mathbb{R}, K=\left(q s_{2}-s_{1}\right)^{-1}, \varepsilon>0$ and $m(u)$ be a moment function of order $1 / K$. Suppose that $\widehat{u}(t, z) \in \mathcal{O}_{1 / \gamma}(D)[[t]]$ is the unique formal power series solution of the Cauchy problem (11) and

$$
\varphi(z) \in \mathcal{O}_{1 / \gamma}^{q K}\left(\bigcup_{l=0}^{q \gamma-1} \widehat{S}_{\left(d+\arg \lambda_{0}+2 l \pi\right) / q}(\varepsilon / q)\right) .
$$

Then, $\widehat{u}(t, z)$ is $K$-summable in the direction $d$ and for every $\tilde{d} \in\left(d-\frac{\varepsilon}{2}, d+\frac{\varepsilon}{2}\right)$ and for every $\tilde{\varepsilon} \in(0, \varepsilon)$, there exists $r>0$ such that its $K$-sum $u^{\tilde{d}} \in \mathcal{O}_{1,1 / \gamma}\left(S_{\tilde{d}}(\pi / K-\tilde{\varepsilon}, r) \times D\right)$ is given by

$$
u^{\tilde{d}}(t, z)=\mathcal{S}_{K, \tilde{d}} \widehat{u}(t, z)=\left(T_{m, \tilde{d}} v\right)(t, z)=\int_{e^{i d \mathbb{R}_{+}}} e_{m}(s / t) v(s, z) \frac{d s}{s},
$$

where $v(t, z)=\widehat{\mathcal{B}}_{m} \widehat{u}(t, z)$ has the integral representation

$$
\begin{aligned}
& v(t, z)=\frac{t^{\beta-1}}{(\beta-1) !} \partial_{t}^{\beta-1} \frac{1}{2 \gamma \pi i} \oint_{|w|=\varepsilon}^{\gamma} \varphi(w) \int_{r_{0} e^{i \theta}}^{\infty e^{i \theta}} \\
& E_{\bar{m}_{1}}(t \lambda(\zeta)) E_{\tilde{m}_{2}}\left(\zeta^{1 / \gamma} z^{1 / \gamma}\right) \frac{e_{m_{2}}(\zeta w)}{\zeta w} d \zeta d w
\end{aligned}
$$

with $\bar{m}_{1}(u)=m_{1}(u) m(u), \tilde{m}_{2}(u)=m_{2}(u / \gamma)$ and $\theta \in\left(-\arg w-\frac{s_{2} \pi}{2},-\arg w+\frac{s_{2} \pi}{2}\right)$.

Proof First, observe that by Proposition 4 we get $\widehat{u}(t, z) \in \mathcal{O}_{1 / \gamma}(D)[[t]]_{q s_{2}-s_{1}}$. Hence, the function $v(t, z):=\widehat{\mathcal{B}}_{m} \widehat{u}(t, z)$ belongs to the space $\mathcal{O}_{1,1 / \gamma}\left(D^{2}\right)$. Moreover, by Proposition 3 it satisfies the following:

$$
\left\{\begin{array}{l}
\left(\partial_{\bar{m}_{1}, t}-\lambda\left(\partial_{m_{2}, z}\right)\right)^{\beta} v=0 \\
\partial_{\bar{m}_{1}, t}^{j} v(0, z)=0(j=0, \ldots, \beta-2) \\
\partial_{\bar{m}_{1}, t}^{\beta} v(0, z)=\lambda^{\beta-1}\left(\partial_{m_{2}, z}\right) \varphi(z) \in \mathcal{O}_{1 / \gamma}(D) .
\end{array}\right.
$$

Hence by [16, Lemma 3], we get the integral representation (14) of $v(t, z)$.

Since $\varphi(z)$ satisfies $(12)$, by [16, Lemma 4], we conclude that $v(t, z) \in \mathcal{O}_{1,1 / \gamma}^{K}\left(\widehat{S}_{d}(\varepsilon) \times\right.$ $D)$. So, for every $\tilde{d} \in\left(d-\frac{\varepsilon}{2}, d+\frac{\varepsilon}{2}\right)$, the function $u^{\tilde{d}}(t, z):=T_{m, \tilde{d}} v(t, z)$ is well-defined and by the definitions of kernel functions (Definitions 2 and 3 ) for every $\tilde{\varepsilon} \in(0, \varepsilon)$, there exists $r>0$ such that $u^{\tilde{d}} \in \mathcal{O}_{1,1 / \gamma}\left(S_{\tilde{d}}(\pi / K-\tilde{\varepsilon}, r) \times D\right)$.

Now, we are ready to describe the Stokes phenomenon and the maximal family of solutions of the simple moment pseudodifferential equation (11) with the Cauchy data having the separate singular point at $z_{0} \in \mathbb{C} \backslash\{0\}$.

Theorem 2 Let $\widehat{u}$ be a formal solution of Eq. 11 with $\varphi \in \mathcal{O}_{1 / \gamma}^{q K}\left(\widetilde{\mathbb{C} \backslash\left\{z_{0}\right\}}\right)$ for some $z_{0} \in$ $\mathbb{C} \backslash\{0\}$. Set $K:=\left(q s_{2}-s_{1}\right)^{-1}, \delta_{l}:=q \arg z_{0}+\frac{2 l \pi}{v}-\arg \lambda_{0}$ and $u_{l}:=u^{\tilde{d}}$ for $\tilde{d} \in\left(\delta_{l}, \delta_{l+1}\right)$ $\bmod 2 q \pi\left(\right.$ for $l=0, \ldots, \mu-1$ with $\left.\delta_{\mu}:=\delta_{0}+2 q \pi\right)$, where $u^{\tilde{d}}$ is given by Eq. 13. Finally, let $W_{r}=\{t \in W: 0<|t|<r\}$ for $r>0$, where $W$ is the Riemann surface of the function $t \mapsto t^{\frac{1}{q}}$.

Then, for every $\tilde{\varepsilon}>0$, there exists $r>0$ such that $u_{l} \in \mathcal{O}_{1,1 / \gamma}\left(S_{\delta_{l}+\frac{\pi}{v}}\left(\left(K^{-1}+\frac{2}{v}\right) \pi-\right.\right.$ $\tilde{\varepsilon}, r) \times D)(l=0, \ldots, \mu-1)$ and $\left\{u_{0}, \ldots, u_{\mu-1}\right\}$ is a maximal family of solutions of Eq. 11 on $W_{r} \times D$. 
Moreover, the sets $\mathcal{L}_{\delta_{l}}$ and $\mathcal{L}_{\delta_{l} \pm \frac{\pi}{2 K}}(l=0, \ldots, \mu-1)$ are respectively Stokes lines and anti-Stokes lines for $\widehat{u}$. The jump across the Stokes line $\mathcal{L}_{\delta_{l}}$ is given by the following:

$$
J_{\mathcal{L}_{\delta_{l}}} \widehat{u}(t, 0)=u_{l}(t, 0)-u_{l-1}(t, 0)=u^{\delta_{l}^{+}}(t, 0)-u^{\delta_{l}^{-}}(t, 0)=F_{l}(s, 0)\left[\frac{e_{m}(s / t)}{s}\right],
$$

where $F_{l}(s, 0) \in \mathcal{H}^{q K}\left(\mathcal{L}_{\delta_{l}}\right)$ is defined by $F_{l}(s, 0):=[v(s, 0)]_{\delta_{l}}$ and $v(s, z)=\widehat{\mathcal{B}}_{m} \widehat{u}(s, z)$ has the representation (14).

Proof First observe that, if $d \neq \delta_{l} \bmod 2 q \pi$ for $l=0, \ldots, \mu-1$ then $\varphi$ satisfies the assumption (12) for sufficiently small $\varepsilon>0$. Hence, by Proposition 5, $\widehat{u}$ is $K$-summable in a direction $\tilde{d} \in \mathbb{R}, \tilde{d} \neq \delta_{l} \bmod 2 q \pi$ for $l=0, \ldots, \mu-1$ and its $K$-sum $u^{\tilde{d}}(t, z)$ satisfies (13).

Observe that $u^{\tilde{d}}(t, z)=u^{\tilde{d}}\left(t e^{2 q \pi i}, z\right)$ and $q$ is the smallest positive rational number for which this equality holds. Moreover, the set of singular directions of $\widehat{u}(t, z)$ modulo $2 q \pi$ is given by $\left\{\delta_{l} \bmod 2 q \pi: l=0, \ldots, \mu-1\right\}$. Hence by [18, Theorem 3$]$, for every $\tilde{\varepsilon}>0$, there exists $r>0$ such that $\left\{u_{0}, \ldots, u_{\mu-1}\right\}$ with $u_{l} \in \mathcal{O}_{1,1 / \gamma}\left(S_{\delta_{l}+\frac{\pi}{v}}\left(\left(K^{-1}+\frac{2}{v}\right) \pi-\tilde{\varepsilon}, r\right) \times\right.$ D) $(l=0, \ldots, \mu-1)$ is a maximal family of solutions of Eq. 11. Moreover, Stokes lines for $\widehat{u}$ are the sets $\mathcal{L}_{\delta_{l}}$ and anti-Stokes lines for $\widehat{u}$ are the sets $\mathcal{L}_{\delta_{l} \pm \frac{\pi}{2 K}}$.

Now, we are ready to calculate the jump across Stokes line $\mathcal{L}_{\delta_{l}}$.

$$
\begin{aligned}
J_{\mathcal{L}_{\delta_{l}}} \widehat{u}(t, z)= & u^{\delta_{l}^{+}}(t, z)-u^{\delta_{l}^{-}}(t, z) \stackrel{(13)}{=} \int_{e^{i \delta_{l}^{+}}} e_{\mathbb{R}_{+}}(s / t) v(s, z) \frac{\mathrm{ds}}{s} \\
& -\int_{e^{i \delta_{l}^{-}} \mathbb{R}_{+}} e_{m}(s / t) v(s, z) \frac{\mathrm{ds}}{s} .
\end{aligned}
$$

Hence,

$$
J_{\mathcal{L}_{\delta_{l}}} \widehat{u}(t, 0)=F_{l}(s, 0)\left[\frac{e_{m}(s / t)}{s}\right],
$$

where $F_{l}(s, 0) \in \mathcal{H}^{q K}\left(\mathcal{L}_{\delta_{l}}\right)$ is a hyperfunction on $\mathcal{L}_{\delta_{l}}$ defined by $F_{l}(s, 0):=[v(s, 0)]_{\delta_{l}}$.

Now, we return to the general equation (8). For convenience, we assume that

$$
P(\lambda, \zeta)=P_{0}(\zeta) \tilde{P}(\lambda, \zeta)=P_{0}(\zeta) \prod_{i=1}^{\tilde{n}} \prod_{\alpha=1}^{l_{i}}\left(\lambda-\lambda_{i \alpha}(\zeta)\right)^{N_{i \alpha}}
$$

where $\lambda_{i \alpha}(\zeta) \sim \lambda_{i \alpha} \zeta^{q_{i}}$ is the root of the characteristic equation with $q_{i} \in \mathbb{Q}$ and $\lambda_{i \alpha} \in$ $\mathbb{C} \backslash\{0\}$ for $i=1, \ldots, \tilde{n}$ and $\alpha=1, \ldots, l_{i}$. Without loss of generality, we may assume that there exist exactly $n, n \leq \tilde{n}$, pole orders $q_{i}$, which are greater than $s_{1} / s_{2}$, where $s_{1}, s_{2}$ are orders of moment functions $m_{1}, m_{2}$ respectively. We also assume that $s_{1} / s_{2}<q_{1}<\cdots<$ $q_{n}<\infty$ and let $K_{i}:=\left(q_{i} s_{2}-s_{1}\right)^{-1}$ for $i=1, \ldots, n$. Under the above conditions, we have

Theorem 3 Let $\widehat{u}$ be a normalised formal solution of Eq. 8, $z_{0} \in \mathbb{C} \backslash\{0\}$ and $\varphi_{j}(z) \in$ $\mathcal{O}^{q_{n} K_{n}}\left(\widehat{\mathbb{C} \backslash\left\{z_{0}\right\}}\right)$ for $j=0, \ldots, N-1$. Let $Q:=\frac{L C M\left(\mu_{1}, \ldots, \mu_{n}\right)}{G C D\left(v_{1}, \ldots, v_{n}\right)}$ and let

$$
\Lambda_{i}:=\left\{\delta: \delta=q_{i} \arg z_{0}+\frac{2 j \pi}{\nu_{i}}-\arg \lambda_{i \alpha} \bmod 2 Q \pi, 0 \leq j \leq Q v_{i}-1,1 \leq \alpha \leq l_{i}\right\}
$$


for $i=1, \ldots, n$. It means that we may assume that there exist $0 \leq \delta_{i, 1}<\cdots<\delta_{i, n_{i}}<$ $2 Q \pi$ such that $\Lambda_{i}=\left\{\delta_{i, 1}, \ldots, \delta_{i, n_{i}}\right\}$. Moreover, let

$$
I_{i, j}:=\left(\delta_{i, j}-\frac{\pi}{2 K_{i}}, \delta_{i, j+1}+\frac{\pi}{2 K_{i}}\right) \quad \text { for } \quad j=1, \ldots, n_{i}, \quad i=1, \ldots, n,
$$

with $\delta_{i, n_{i}+1}:=\delta_{i, 1}+2 Q \pi$,

$$
\mathcal{J}:=\left\{\mathbf{l}=\left(l_{1}, \ldots, l_{n}\right) \in \mathbb{N}^{n}: 1 \leq l_{i} \leq n_{i},\left|I_{i, l_{i}} \cap \cdots \cap I_{n, l_{n}}\right|>\frac{\pi}{K_{i}} \text { for } i=1, \ldots, n\right\},
$$

$\mathbf{K}:=\left(K_{1}, \ldots, K_{n}\right)$ and $W_{r}=\{t \in W: 0<|t|<r\}$ for $r>0$, where $W$ is the Riemann surface of the function $t \mapsto t^{1 / Q}$.

Then, the following conditions holds:

(a) The formal solution $\widehat{u}$ is $\mathbf{K}$-multisummable, $\widehat{u}=\widehat{u}_{0}+\widehat{u}_{1}+\cdots+\widehat{u}_{n}$, where $\widehat{u}_{0}$ is a convergent power series solution of

$$
\left(\prod_{i=n+1}^{\tilde{n}} \prod_{\alpha=1}^{l_{i}}\left(\partial_{m_{1}, t}-\lambda_{i \alpha}\left(\partial_{m_{2}, z}\right)\right)^{N_{i \alpha}}\right) u_{0}=0
$$

and $\widehat{u}_{i}$ is a $K_{i}$-summable power series solution of

$$
\left(\prod_{\alpha=1}^{l_{i}}\left(\partial_{m_{1}, t}-\lambda_{i \alpha}\left(\partial_{m_{2}, z}\right)\right)^{N_{i \alpha}}\right) u_{i}=0 \quad \text { for } \quad i=1, \ldots, n .
$$

Moreover,

$$
\mathcal{S}_{\mathbf{K}, \mathbf{d}} \widehat{u}=u_{0}+\mathcal{S}_{K_{1}, d_{1}} \widehat{u}_{1}+\cdots+\mathcal{S}_{K_{n}, d_{n}} \widehat{u}_{n}
$$

for any admissible nonsingular multidirection $\mathbf{d}=\left(d_{1}, \ldots, d_{n}\right)$.

(b) For every $\mathbf{l} \in \mathcal{J}$, the function $u_{\mathbf{l}}(t, z):=\mathcal{S}_{\mathbf{K}, \mathbf{d}} \widehat{u}$ is a well-defined actual solution of Eq. 8, where $\mathbf{d}$ is an admissible nonsingular multidirection satisfying $d_{i} \in$ $\left(\delta_{i, l_{i}}, \delta_{i, l_{i}+1}\right)$ for $i=1, \ldots, n$,.

(c) For every $\varepsilon>0$, there exists $r>0$ such that $u_{\mathbf{l}} \in \mathcal{O}_{1,1 / \gamma}\left(V_{\mathbf{l}}(\varepsilon, r) \times D\right)$, where

$$
V_{\mathbf{l}}(\varepsilon, r):=\left\{t \in W_{r}:\left(\arg t-\frac{\varepsilon}{2}, \arg t+\frac{\varepsilon}{2}\right) \subseteq I_{1, l_{1}} \cap \cdots \cap I_{n, l_{n}}\right\} .
$$

(d) $\left\{u_{\mathbf{l}}\right\}_{\mathbf{l} \in \mathcal{J}}$ is a maximal family of solutions of Eq. 8 .

(e) For every $i \in\{1, \ldots, n\}$, the sets $\mathcal{L}_{\delta_{i, j}}$ (resp. $\mathcal{L}_{\delta_{i, j} \pm \frac{\pi}{2 K_{i}}}$ ), $j=1, \ldots, n_{i}$ are Stokes lines (resp. anti-Stokes lines) of level $K_{i}$.

(f) For every $i \in\{1, \ldots, n\}$ and $j \in\left\{1, \ldots, n_{i}\right\}$, the jump across the Stokes line $\mathcal{L}_{\delta_{i, j}}$ of level $K_{i}$ is given by

$$
J_{\mathcal{L}_{\delta_{i, j}}, K_{i}} \widehat{u}(t, 0)=u_{\mathbf{l}}(t, 0)-u_{\mathbf{I}^{\prime}}(t, 0)=u_{i}^{\delta_{i, j}^{+}}(t, 0)-u_{i}^{\delta_{i, j}^{-}}(t, 0)=F_{i, j}(s, 0)\left[\frac{e_{\bar{m}_{i}}(s / t)}{s}\right],
$$

where $u_{i}^{\delta_{i, j}^{ \pm}}=\mathcal{S}_{K_{i}, \delta_{i, j}^{ \pm}} \widehat{u}_{i}, \bar{m}_{i}$ is a moment function of order $1 / K_{i}, F_{i, j}(s, 0)$ is a hyperfunction on $\mathcal{L}_{\delta_{i, j}}$ defined by $F_{i, j}(s, 0):=\left[v_{i}(s, 0)\right]_{\delta_{i, j}}, v_{i}(s, z):=\widehat{\mathcal{B}}_{\bar{m}_{i}} \widehat{u}_{i}(s, z)$ and $\mathbf{l}, \mathbf{l}^{\prime} \in \mathcal{J}$ satisfy $l_{i}^{\prime}=j-1$ in the case when $l_{i}=j$ and $j>1, l_{i}^{\prime}=n_{i}$ in the case when $l_{i}=1$, and $l_{\alpha}=l_{\alpha}^{\prime}$ for $\alpha \neq i$. 
Moreover, under the additional condition that $\widehat{u}$ is a normalised formal solution of Eq. 8 with $m_{2}(u)=\Gamma(1+u)$ (i.e. when $\widehat{u}$ is a normalised formal solution of the Cauchy problem $P\left(\partial_{m_{1}, t}, \partial_{z}\right) u=0, \partial_{m_{1}, t}^{j} u(0, z)=\varphi_{j}(z)$ for $\left.j=0, \ldots, N-1\right)$, we may replace the assertion $(f)$ by

(f') For every $i \in\{1, \ldots, n\}, j \in\left\{1, \ldots, n_{i}\right\}$ and $z \in D$, the jump across the Stokes line $\mathcal{L}_{\delta_{i, j}}$ of level $K_{i}$ is given by the following.

$$
J_{\mathcal{L}_{\delta_{i, j}}, K_{i}} \widehat{u}(t, z)=u_{\mathbf{I}}(t, z)-u_{\mathbf{I}^{\prime}}(t, z)=u_{i}^{\delta_{i, j}^{+}}(t, z)-u_{i}^{\delta_{i, j}^{-}}(t, z)=F_{i, j}(s, z)\left[\frac{e_{\bar{m}_{i}}(s / t)}{s}\right],
$$

where $u_{i}^{\delta_{i, j}^{ \pm}}=\mathcal{S}_{K_{i}, \delta_{i, j}^{ \pm}} \widehat{u}_{i}, \bar{m}_{i}$ is a moment function of order $1 / K_{i}, F_{i, j}(s, z)$ is a hyperfunction on $\mathcal{L}_{\delta_{i, j}(z)}$ defined by $F_{i, j}(s, z):=\left[v_{i}(s, z)\right]_{\delta_{i, j}(z)}, \delta_{i, j}(z):=\delta_{i, j}+$ $q_{i}\left(\arg \left(z_{0}-z\right)-\arg z_{0}\right), v_{i}(s, z):=\widehat{\mathcal{B}}_{\bar{m}_{i}} \widehat{u}_{i}(s, z)$ and $\mathbf{l}, \mathbf{l}^{\prime} \in \mathcal{J}$ satisfy the same conditions as in $(f)$.

Proof Since $P(\lambda, \zeta)$ is given by Eq. 15, by Proposition 4, a normalised formal solution $\widehat{u}$ of Eq. 8 may be written as $\widehat{u}=\widehat{u}_{0}+\widehat{u}_{1}+\cdots+\widehat{u}_{n}$, where $\widehat{u}_{0}$ is a convergent power series solution of the pseudodifferential equation (16) and $\widehat{u}_{i}$ is a $1 / K_{i}$-Gevrey power series solution of Eq. 17 with the initial data having the same holomorphic properties as $\varphi_{j}(z)$. More precisely, by Proposition 4 we conclude that $\widehat{u}_{i}=\sum_{\alpha=1}^{l_{i}} \sum_{\beta=1}^{N_{i \alpha}} \widehat{u}_{i \alpha \beta}$, where $\widehat{u}_{i \alpha \beta}$ is a formal solution of a simple pseudodifferential equation as follows:

$$
\left\{\begin{array}{l}
\left(\partial_{m_{1}, t}-\lambda_{i \alpha}\left(\partial_{m_{2}, z}\right)\right)^{\beta} u_{i \alpha \beta}=0 \\
\partial_{m_{1}, t}^{j} u_{i \alpha \beta}(0, z)=0(j=0, \ldots, \beta-2) \\
\partial_{m_{1}, t}^{\beta-1} \widehat{u}_{i \alpha \beta}(0, z)=\lambda_{i \alpha}^{\beta-1}\left(\partial_{m_{2}, z}\right) \varphi_{i \alpha \beta}(z)
\end{array}\right.
$$

where $\varphi_{i \alpha \beta}(z):=\sum_{j=0}^{N-1} d_{i \alpha \beta j}\left(\partial_{m_{2}, z}\right) \varphi_{j}(z) \in \mathcal{O}_{1 / \gamma}(D)$ and $d_{i \alpha \beta j}(\zeta)$ are some holomorphic functions of the variable $\xi=\zeta^{1 / \gamma}$ and of polynomial growth. Since $\varphi_{j}(z) \in \mathcal{O}^{q_{n} K_{n}}\left(\widetilde{\mathbb{C} \backslash\left\{z_{0}\right\}}\right)$ and $q_{i} K_{i} \leq q_{n} K_{n}$, we see that $\varphi_{i \alpha \beta}(z) \in$ $\mathcal{O}_{1 / \gamma}^{q_{i} K_{i}}\left(\bigcup_{l=0}^{q_{i} \gamma-1} \widehat{S}_{\left(d+\arg \lambda_{\alpha}+2 l \pi\right) / q_{i}}\right)$ for every direction $d \neq q_{i} \arg z_{0}+\frac{2 j \pi}{v_{i}}-\arg \lambda_{i \alpha}$ $\bmod 2 \pi q_{i}, j=0, \ldots, \mu-1$.

Hence, by Proposition 5 and Theorem 2, we see that $\widehat{u}_{i \alpha \beta}$ is $K_{i}$-summable with the singular directions given by $q_{i} \arg z_{0}+\frac{2 j \pi}{v_{i}}-\arg \lambda_{i \alpha} \bmod 2 \pi q_{i}$ for $j=0, \ldots \mu_{i}-1$. Consequently, $\widehat{u}$ is $\mathbf{K}$-multisummable in any nonsingular admissible multidirection $\mathbf{d}=$ $\left(d_{1}, \ldots, d_{n}\right)$. Since a formal power series $\widehat{u}_{0}$ is convergent, its sum $u_{0}$ is well-defined and by Remark 7, we conclude that $\mathbf{K}$-multisum $\mathcal{S}_{\mathbf{K}, \mathbf{d}} \widehat{d}$ of $\widehat{u}$ is given by Eq. 18, so (a) holds.

Since $\widehat{u}$ is $K$-multisummable, using Theorem 1 we conclude that (b), (c), and (d) hold.

Since the set of singular directions of order $K_{i}$ is given by $\Lambda_{i}$, we get the description of Stokes lines $\mathcal{L}_{\delta_{i, j}}$ and anti-Stokes lines $\mathcal{L}_{\delta_{i, j} \pm \frac{\pi}{2 K_{i}}}$ of level $K_{i}$ for $\delta_{i, j} \in \Lambda_{i}$ and $i=1, \ldots, n$, so (e) is also satisfied.

Finally, to obtain (f) by Theorem 2, we calculate the jumps for $\widehat{u}$ across the Stokes lines $\mathcal{L}_{d_{i}}$ of level $K_{i}$. Using Remark 9, we get (f').

Let us illustrate our theory on the following simple example. 
Example 4 Let $\widehat{u}$ be a formal solution of the Cauchy problem

$$
\left\{\begin{array}{l}
\left(\partial_{t}-\partial_{z}^{2}\right)\left(\partial_{t}-\partial_{z}^{3}\right) \widehat{u}=0 \\
u(0, z)=\varphi_{1}(z) \in \mathcal{O}^{3 / 2}\left(\widehat{\mathbb{C} \backslash\left\{z_{0}\right\}}\right), \\
\partial_{t} u(0, z)=\varphi_{2}(z) \in \mathcal{O}^{3 / 2}\left(\widehat{\mathbb{C} \backslash\left\{z_{0}\right\}}\right),
\end{array}\right.
$$

where $\arg z_{0} \in\left(-\frac{\pi}{2}, \frac{\pi}{2}\right)$.

Then by Proposition 4 (see also [16, Theorem 1]), $\widehat{u}=\widehat{u}_{1}+\widehat{u}_{2}$, where $\widehat{u}_{1}, \widehat{u}_{2}$ are formal solutions of the following:

$$
\begin{aligned}
& \left(\partial_{t}-\partial_{z}^{2}\right) u_{1}=0, \quad u_{1}(0, z)=c_{11}\left(\partial_{z}\right) \varphi_{1}(z)+c_{12}\left(\partial_{z}\right) \varphi_{2}(z)=: \tilde{\varphi}_{1}(z), \\
& \left(\partial_{t}-\partial_{z}^{3}\right) u_{2}=0, \quad u_{2}(0, z)=c_{21}\left(\partial_{z}\right) \varphi_{1}(z)+c_{22}\left(\partial_{z}\right) \varphi_{2}(z)=: \tilde{\varphi}_{2}(z),
\end{aligned}
$$

where $c_{\mathrm{ij}}\left(\partial_{z}\right), i, j=1,2$, are pseudodifferential operators defined by $c_{11}(\zeta):=\frac{\zeta}{\zeta-1}$, $c_{12}(\zeta):=\frac{1}{\zeta^{2}-\zeta^{3}}, c_{21}(\zeta):=\frac{1}{1-\zeta}$ and $c_{22}(\zeta):=\frac{1}{\zeta^{3}-\zeta^{2}}$.

Now, we are ready to describe the Stokes phenomenon and the maximal family of solutions of Eq. 19. By Theorem 2, $\widehat{u}_{1}$ is 1 -summable with singular directions $d_{1, l}:=$ $2 \arg z_{0}+2 l \pi$ and $\widehat{u}_{2}$ is $1 / 2$-summable with singular directions $d_{2, l}:=3 \arg z_{0}+2 l \pi$ for $l \in \mathbb{Z}$. Hence $\widehat{u}$ is $(1,1 / 2)$-summable, the set of singular directions (modulo $12 \pi$ ) of level 1 is given by $\left\{d_{1,0}, \ldots, d_{1,5}\right)$, and the set of singular directions (modulo $12 \pi$ ) of level $1 / 2$ is given by $\left\{d_{2,0}, \ldots, d_{2,5}\right)$. It means that $\mathcal{L}_{d_{1, l}}$ and $\mathcal{L}_{d_{1, l} \pm \frac{\pi}{2}}$ are respectively Stokes and anti-Stokes lines of level 1, and analogously $\mathcal{L}_{d_{2, l}}$ and $\mathcal{L}_{d_{2, l} \pm \pi}$ are respectively Stokes and anti-Stokes lines of level $1 / 2(l=0, \ldots, 5)$.

Next, let $I_{1, j}:=\left(-\frac{\pi}{2}+2 \pi j+2 \arg z_{0}, \frac{\pi}{2}+2 \pi(j+1)+2 \arg z_{0}\right)$ for $j=0, \ldots, 5$ and $I_{2, k}:=\left(-\pi+2 \pi k+3 \arg z_{0}, \pi+2 \pi(k+1)+3 \arg z_{0}\right)$ for $k=0, \ldots, 5$. Since $\arg z_{0} \in\left(-\frac{\pi}{2}, \frac{\pi}{2}\right)$, we conclude that

$$
\left|I_{1, j} \cap I_{2, k}\right|= \begin{cases}3 \pi & |j-k|=0 \\ \frac{3}{2} \pi \pm \arg z_{0} & j-k= \pm 1 \\ 0 & |j-k|>1\end{cases}
$$

Hence, $\mathcal{J}=\{(j, k): 0 \leq j, k \leq 5,|j-k| \leq 1\}$ and $\left\{u_{(j, k)}\right\}_{(j, k) \in \mathcal{J}}$ is a maximal family of solutions of Eq. 19 on the Riemann surface of $t \mapsto t^{\frac{1}{6}}$, where $u_{(j, k)}:=u_{1, j}+u_{2, k}$, $u_{1, j}:=u_{1}^{d}=\mathcal{S}_{1, d} \widehat{u}_{1}$ for $d \in\left(d_{1, j}, d_{1, j+1}\right)=\left(2 \arg z_{0}+2 \pi j, 2 \arg z_{0}+2 \pi(j+1)\right)$ and $u_{2, k}:=u_{2}^{d}=\mathcal{S}_{2, k} \widehat{u}_{2}$ for $d \in\left(d_{2, j}, d_{2, j+1}\right)=\left(3 \arg z_{0}+2 \pi k, 3 \arg z_{0}+2 \pi(k+1)\right)$.

Using [18] and [22], we are also able to calculate the jumps across the Stokes lines. Namely,

$$
\begin{aligned}
J_{\mathcal{L}_{d_{1, j}, 1} \widehat{u}(t, z)} & =u_{(j, j)}(t, z)-u_{(j-1, j)}(t, z)=u_{1, j}(t, z)-u_{1, j-1}(t, z) \\
& =J_{\mathcal{L}_{d_{1, j}}} \widehat{u}_{1}=u_{1}^{d_{1, j}^{+}}(t, z)-u_{1}^{d_{1, j}^{-}}(t, z)=F_{1, z}(s)\left[\frac{1}{\sqrt{4 \pi t}} e^{-\frac{s^{2}}{4 t}}\right],
\end{aligned}
$$

where $F_{1, z}(s):=\left[\tilde{\varphi}_{1}(z+s)\right] \arg \left(z_{0}-z\right)$ is a hyperfunction on $\left\{s \in \tilde{\mathbb{C}}: \arg s=\arg \left(z_{0}-z\right)\right\}$. Analogously,

$$
\begin{aligned}
J_{\mathcal{L}_{d_{2, j}, 1 / 2} \widehat{u}(t, z)} & =u_{(j, j)}(t, z)-u_{(j, j-1)}(t, z)=u_{2, j}(t, z)-u_{2, j-1}(t, z) \\
& =J_{\mathcal{L}_{d_{1, j}}} \widehat{u}_{2}=u_{2, j}^{d_{2, j}^{+}}(t, z)-u_{2}^{d_{2, j}^{-}}(t, z)=F_{2, z}(s)\left[\frac{1}{3 \sqrt[3]{t}} C_{3}(s / \sqrt[3]{t})\right],
\end{aligned}
$$


where $F_{2, z}(s):=\left[\tilde{\varphi}_{2}(z+s)\right] \arg \left(z_{0}-z\right)$ is a hyperfunction on $\left\{s \in \tilde{\mathbb{C}}: \arg s=\arg \left(z_{0}-z\right)\right\}$ and $C_{3}(\tau)$ is the Ecalle kernel defined by $C_{3}(\tau):=\sum_{n=0}^{\infty} \frac{(-\tau)^{n}}{n ! \Gamma\left(1-\frac{n+1}{3}\right)}$.

\section{Moment Partial Differential Equations-Special Cases}

In this section, we will consider certain special cases of moment partial differential equations. We derive Stokes lines and jumps across these Stokes lines in terms of hyperfunctions.

Case 1. Let us consider the following equation as follows:

$$
\left\{\begin{array}{l}
\partial_{m_{1}, t}^{p} u(t, z)=\partial_{z}^{q} u(t, z) \text { with } 0<\mathrm{ps}_{1}<q \\
u(0, z)=\varphi(z), \\
\partial_{m_{1}, t}^{j} u(0, z)=0, \text { for } j=1,2, \ldots, p-1
\end{array}\right.
$$

with $\varphi(z) \in \mathcal{O}^{\frac{q}{q-\mathrm{ps}_{1}}}\left(\widetilde{\mathbb{C} \backslash\left\{z_{0}\right\}}\right)$ for some $z_{0} \in \mathbb{C} \backslash\{0\}$; where $m_{1}$ is a moment function of order $s_{1}>0$ corresponding to a kernel function $e_{m_{1}}(z)$ of order $1 / s_{1}$.

The above Cauchy problem has a unique formal solution as follows:

$$
\widehat{u}(t, z)=\sum_{n=0}^{\infty} \frac{\varphi^{(\mathrm{qn})}(z)}{m_{1}(\mathrm{pn})} t^{p n}
$$

to which we first apply the $m$-moment Borel transform. We obtain the following:

$$
\begin{aligned}
\left(\widehat{\mathcal{B}}_{m} \widehat{u}\right)(t, z) & =\sum_{n=0}^{\infty} \frac{\varphi^{(\mathrm{qn})}(z)}{m_{1}(\mathrm{pn})} \cdot \frac{m_{1}(\mathrm{pn})}{\Gamma\left(1+\frac{q}{p} \cdot \mathrm{pn}\right.} t^{\left(\frac{p}{q}\right) \mathrm{qn}}=\sum_{n=0}^{\infty} \frac{\varphi^{(\mathrm{qn})}(z)}{(\mathrm{qn})} t^{\left(\frac{p}{q}\right) \mathrm{qn}} \\
& =\frac{1}{q}\left(\varphi\left(z+\sqrt[q]{t^{p}}\right)+\varphi\left(z+e^{\frac{2 \pi i}{q}} \sqrt[q]{t^{p}}\right)+\cdots+\varphi\left(z+e^{\frac{2(q-1) \pi i}{q}} \sqrt[q]{t^{p}}\right)\right)
\end{aligned}
$$

where $m(n):=\frac{\Gamma\left(1+\frac{q}{p} n\right)}{m_{1}(n)}=\frac{\Gamma \frac{q}{p}(n)}{m_{1}(n)}$ is a moment function of order $\frac{q}{p}-s_{1}$ corresponding to a kernel function $e_{m}(z)$ of order $\frac{p}{q-\mathrm{ps}_{1}}$.

Let $f(s, z):=\left(\widehat{\mathcal{B}}_{m} \widehat{u}\right)(s, z)$, then by using $m$-moment Laplace transform in a nonsingular direction $d$ we get the following:

$$
\begin{aligned}
\left(T_{m, d} f\right)(t, z)= & \int_{e^{\mathrm{id} \mathbb{R}_{+}}} e_{m}(s / t) f(s, z) \frac{\mathrm{ds}}{s} \\
= & \frac{1}{q} \int_{e^{\mathrm{id}} \mathbb{R}_{+}} e_{m}(s / t)\left(\varphi\left(z+\sqrt[q]{s^{p}}\right)+\varphi\left(z+e^{\left.\frac{2 \pi i}{q} \sqrt[q]{s^{p}}\right)+\ldots}\right.\right. \\
& \left.+\varphi\left(z+e^{\frac{2(q-1) \pi i}{q}} \sqrt[q]{s^{p}}\right)\right) \frac{\mathrm{ds}}{s}
\end{aligned}
$$


Thus, by Proposition 5, the unique formal solution $\widehat{u}(t, z)$ of this Cauchy problem is $\frac{p}{q-\mathrm{ps}_{1}}$-summable in the direction $d$, and for every $\varepsilon>0$, there exists $r>0$ such that its $\frac{p}{q-\mathrm{ps}_{1}}$-sum $u \in \mathcal{O}\left(S_{d}\left(\frac{\pi\left(q-\mathrm{ps}_{1}\right)}{p}-\varepsilon, r\right) \times D\right)$ is given by the following:

$$
\begin{aligned}
u(t, z)= & u^{d}(t, z) \\
= & \frac{1}{q} \int_{e^{i d} \mathbb{R}_{+}} e_{m}(s / t)\left(\varphi\left(z+\sqrt[q]{s^{p}}\right)+\varphi\left(z+e^{\frac{2 \pi i}{q}} \sqrt[q]{s^{p}}\right)+\ldots\right. \\
& \left.+\varphi\left(z+e^{\frac{2(q-1) \pi i}{q}} \sqrt[q]{s^{p}}\right)\right) \frac{\mathrm{ds}}{s}
\end{aligned}
$$

Let $\theta:=\arg z_{0}, \delta:=\frac{q \theta}{p}$. Then, $\mathcal{L}_{\delta+\frac{2 \pi j}{p}}(j=0,1, \ldots, p-1)$ are Stokes lines for $\widehat{u}$. For every sufficiently small $\varepsilon>0$, there exists $r>0$ such that for every fixed $z \in D_{r}$ the jump is given by the following:

$$
\begin{aligned}
J_{\mathcal{L}_{\delta} \widehat{u}}(t, z)= & u^{\delta+\varepsilon}(t, z)-u^{\delta-\varepsilon}(t, z)=F_{z}(s)\left[\frac{e_{m}(s / t)}{s}\right] \\
= & {\left[\varphi\left(z+\sqrt[q]{s^{p}}\right)+\varphi\left(z+e^{\frac{2 \pi i}{q}} \sqrt[q]{s^{p}}\right)+\ldots\right.} \\
& \left.+\varphi\left(z+e^{\frac{2(q-1) \pi i}{q}} \sqrt[q]{s^{p}}\right)\right]_{q \frac{\theta_{z}}{p}}\left[\frac{e_{m}(s / t)}{\mathrm{qs}}\right] \\
= & {\left[\varphi\left(z+\sqrt[q]{s^{p}}\right)\right]_{q \frac{\theta_{z}}{p}}\left[\frac{e_{m}(s / t)}{\mathrm{qs}}\right] }
\end{aligned}
$$

with $\theta_{z}=\arg \left(z_{0}-z\right)$. The last equality arising from the fact that in this case all singular points appear in the function $s \mapsto \varphi\left(z+\sqrt[q]{s^{p}}\right)$.

Observe that from [2, Theorem 32] one can derive the function

$$
e_{m}(u)=T_{m_{1}, d}^{-}\left(e_{m_{2}}(1 / z)\right)(1 / u)=-\frac{1}{2 \pi i} \int_{\gamma(d)} E_{m_{1}}\left(\frac{1}{u z}\right) \frac{p}{q}\left(\frac{1}{z}\right)^{\frac{p}{q}} e^{-\left(\frac{1}{z}\right)^{\frac{p}{q}}} \frac{\mathrm{dz}}{z},
$$

where $E_{m_{1}}\left(\frac{1}{u z}\right)=\sum_{n=0}^{\infty} \frac{\left(\frac{1}{u z}\right)^{n}}{m_{1}(n)}, m_{2}(n)=\Gamma\left(1+\frac{q}{p} n\right)$ and, by Example $1, e_{m_{2}}(z)=$ $\frac{p}{q} z^{\frac{p}{q}} e^{-z^{\frac{p}{q}}}$.

Case 2. Let us now study the formal solution

$$
\widehat{u}(t, z)=\sum_{n=0}^{\infty} \frac{\partial_{m_{2}, z}^{n} \varphi(z)}{m_{1}(n)} t^{n}
$$

of the following equation

$$
\left\{\begin{array}{l}
\partial_{m_{1}, t} u(t, z)=\partial_{m_{2}, z} u(t, z) \\
u(0, z)=\varphi(z)
\end{array}\right.
$$

with $\varphi(z) \in \mathcal{O}^{\frac{1}{s_{2}-s_{1}}}\left(\widetilde{\mathbb{C} \backslash\left\{z_{0}\right\}}\right)$ for some $z_{0} \in \mathbb{C} \backslash\{0\}$; where $m_{1}$ is a moment function of order $s_{1}>0$ corresponding to a kernel function $e_{m_{1}}(z)$ of order $1 / s_{1}, m_{2}$ is a moment function of order $s_{2}>0$ corresponding to a kernel function $e_{m_{2}}(z)$ of order $1 / s_{2}$ and $s_{2}>$ $s_{1}$. First, we apply to $\widehat{u}(t, z)$ the $m$-moment Borel transform

$$
\left(\widehat{\mathcal{B}}_{m} \widehat{u}\right)(t, z)=\widehat{\mathcal{B}}_{m}\left(\sum_{n=0}^{\infty} \frac{\partial_{m_{2}, z}^{n} \varphi(z)}{m_{1}(n)} t^{n}\right)=\sum_{n=0}^{\infty} \frac{\partial_{m_{2}, z}^{n} \varphi(z)}{m_{2}(n)} t^{n},
$$


where $m(n):=m_{2}(n) / m_{1}(n)$ is a moment function of order $s_{2}-s_{1}$ corresponding to a kernel function $e_{m}(z)$ of order $k:=\frac{1}{s_{2}-s_{1}}$.

Using [15, Proposition 3], we see that for $|z|<\varepsilon<r$ and $n \in \mathbb{N}$, we have

$$
\partial_{m_{2}, z}^{n} \varphi(z)=\frac{1}{2 \pi i} \oint_{|w|=\varepsilon} \varphi(w) \int_{0}^{\infty(\psi)} \zeta^{n} E_{m_{2}}(z \zeta) \frac{e_{m_{2}}(w \zeta)}{w \zeta} d \zeta d w,
$$

where $\psi \in\left(-\arg w-\frac{\pi s_{2}}{2},-\arg w+\frac{\pi s_{2}}{2}\right)$. Thus,

$$
\begin{aligned}
\left(\widehat{\mathcal{B}}_{m} \widehat{u}\right)(t, z)= & \sum_{n=0}^{\infty} \frac{\partial_{m_{2}, z}^{n} \varphi(z) t^{n}}{m_{2}(n)}=\sum_{n=0}^{\infty} \frac{1}{2 \pi i} \oint_{|w|=\varepsilon} \varphi(w) \int_{0}^{\infty(\psi)} \frac{\zeta^{n} t^{n}}{m_{2}(n)} \\
& E_{m_{2}}(z \zeta) \frac{e_{m_{2}}(w \zeta)}{w \zeta} d \zeta d w \\
= & \frac{1}{2 \pi i} \oint_{|w|=\varepsilon} \varphi(w) \int_{0}^{\infty(\psi)} E_{m_{2}}(t \zeta) E_{m_{2}}(z \zeta) \frac{e_{m_{2}}(w \zeta)}{w \zeta} d \zeta d w
\end{aligned}
$$

Let $f(s, z):=\left(\widehat{\mathcal{B}}_{m} \widehat{u}\right)(s, z)$, then by using $m$-moment Laplace transform in a nonsingular direction $d$, we get the following:

$$
\begin{aligned}
\left(T_{m, d} f\right)(t, z)= & \int_{e^{\mathrm{id} \mathbb{R}_{+}}} e_{m}(s / t) f(s, z) \frac{\mathrm{ds}}{s} \\
= & \int_{e^{\mathrm{id} \mathbb{R}_{+}}} e_{m}(s / t)\left(\frac{1}{2 \pi i} \oint_{|w|=\varepsilon} \varphi(w) \int_{0}^{\infty(\psi)}\right. \\
& \left.E_{m_{2}}(s \zeta) E_{m_{2}}(z \zeta) \frac{e_{m_{2}}(w \zeta)}{w \zeta} d \zeta d w\right) \frac{\mathrm{ds}}{s}
\end{aligned}
$$

Notice that, by [2, Theorem 32], the function $e_{m}(u)$ is of the form

$$
e_{m}(u)=T_{m_{1}, d}^{-}\left(e_{m_{2}}(1 / z)\right)(1 / u)=-\frac{1}{2 \pi i} \int_{\gamma(d)} E_{m_{1}}\left(\frac{1}{\mathrm{uz}}\right) e_{m_{2}}(1 / z) \frac{\mathrm{dz}}{z},
$$

where $E_{m_{1}}\left(\frac{1}{\mathrm{uz}}\right)=\sum_{n=0}^{\infty} \frac{\left(\frac{1}{u z}\right)^{n}}{m_{1}(n)}$.

Thus, by Proposition 5, the unique formal solution $\widehat{u}(t, z)$ of this Cauchy problem is $k$ summable in the direction $d$ and for every $\varepsilon>0$, there exists $r>0$ such that its $k$-sum $u \in \mathcal{O}\left(S_{d}\left(\frac{\pi}{k}-\varepsilon, r\right) \times D\right)$ is given by the following:

$$
\begin{aligned}
u(t, z) & =u^{d}(t, z) \\
& =\int_{e^{\mathrm{id} \mathbb{R}_{+}}} e_{m}(s / t)\left(\frac{1}{2 \pi i} \oint_{|w|=\varepsilon} \varphi(w) \int_{0}^{\infty(\psi)} E_{m_{2}}(s \zeta) E_{m_{2}}(z \zeta) \frac{e_{m_{2}}(w \zeta)}{w \zeta} d \zeta d w\right) \frac{\mathrm{ds}}{s} .
\end{aligned}
$$

Then, $\mathcal{L}_{\delta}$, with $\delta=\theta:=\arg z_{0}$, is a Stokes line for $\widehat{u}$. For $z=0$, the jump is given by the following:

$$
\begin{aligned}
J_{\mathcal{L}_{\delta} \widehat{u}(t, 0)} & =u^{\delta^{+}}(t, 0)-u^{\delta^{-}}(t, 0)=F_{0}(s)\left[\frac{e_{m}(s / t)}{s}\right] \\
& =\left[\frac{1}{2 \pi i} \oint_{|w|=\varepsilon} \varphi(w) \int_{0}^{\infty(\psi)} E_{m_{2}}(s \zeta) \frac{e_{m_{2}}(w \zeta)}{w \zeta} d \zeta d w\right]_{\theta}\left[\frac{e_{m}(s / t)}{s}\right] .
\end{aligned}
$$


Using [2, formula (5.15)], one can derive

$$
\int_{0}^{\infty(\psi)} E_{m_{2}}(s \zeta) \frac{e_{m_{2}}(w \zeta)}{w \zeta} d \zeta=\frac{1}{w-s}
$$

hence,

$$
J_{\mathcal{L}_{\delta}} \widehat{u}(t, 0)=\left[\frac{1}{2 \pi i} \oint_{|w|=\varepsilon} \frac{\varphi(w)}{w-s} d w\right]_{\theta}\left[\frac{e_{m}(s / t)}{s}\right]=[\varphi(s)]_{\theta}\left[\frac{e_{m}(s / t)}{s}\right],
$$

where the last equality follows from the Cauchy integral formula.

Case 3. Now, we take the following equation under consideration

$$
\left\{\begin{array}{l}
\partial_{m_{1}, t}^{q} u(t, z)=\partial_{m_{2}, z}^{q} u(t, z) \\
u(0, z)=\varphi(z) \\
\partial_{m_{1}, t}^{j} u(0, z)=0, \text { for } j-1,2, \ldots, q-1
\end{array}\right.
$$

with $\varphi(z) \in \mathcal{O}^{\frac{1}{s_{2}-s_{1}}}\left(\widetilde{\mathbb{C} \backslash\left\{z_{0}\right\}}\right)$ for some $z_{0} \in \mathbb{C} \backslash\{0\}$, where $m_{1}$ is a moment function of order $s_{1}>0$ corresponding to a kernel function $e_{m_{1}}(z)$ of order $1 / s_{1}, m_{2}$ is a moment function of order $s_{2}>0$ corresponding to a kernel function $e_{m_{2}}(z)$ of order $1 / s_{2}$ and $s_{2}>s_{1}$. Observe that since

$$
\partial_{m_{1}, t}^{q}-\partial_{m_{2}, z}^{q}=\left(\partial_{m_{1}, t}-\partial_{m_{2}, z}\right)\left(\partial_{m_{1}, t}-e^{\frac{2 \pi i}{q}} \partial_{m_{2}, z}\right) \cdot \ldots \cdot\left(\partial_{m_{1}, t}-e^{\frac{2 \pi i(q-1)}{q}} \partial_{m_{2}, z}\right),
$$

then, we can write the following:

$$
\widehat{u}(t, z)=\widehat{u}_{0}(t, z)+\widehat{u}_{1}(t, z)+\ldots+\widehat{u}_{q-1}(t, z),
$$

where, for $j=0,1, \ldots, q-1$

$$
\widehat{u}_{j}(t, z)=\frac{1}{q} \sum_{n=0}^{\infty} \frac{\partial_{m_{2}, z}^{n} \varphi(z)}{m_{1}(n)}\left(e^{\frac{2 \pi \mathrm{ij}}{q}}\right)^{n} t^{n},
$$

is a formal solution of the equation

$$
\left\{\begin{array}{l}
\partial_{m_{1}, t} u_{j}(t, z)=e^{\frac{2 \pi \mathrm{ij}}{q}} \partial_{m_{2}, z} u_{j}(t, z), \\
u_{j}(0, z)=\frac{1}{q} \varphi(z) \in \mathcal{O}^{\frac{1}{s_{2}-s_{1}}}\left(\widetilde{\left.\mathbb{C} \backslash\left\{z_{0}\right\}\right)} .\right.
\end{array}\right.
$$

Notice that, based on reasoning of the case 2 , for each $\widehat{u}_{j}(t, z)$, we obtain that

$$
u_{j}(t, z)=\int_{e^{\mathrm{id} \mathbb{R}_{+}}} e_{m}(s / t)\left(\frac{1}{2 \pi i} \oint_{|w|=\varepsilon} \varphi(w) \int_{0}^{\infty(\psi)} E_{m_{2}}\left(s e^{\frac{2 \pi i j}{q}} \zeta\right) E_{m_{2}}(z \zeta) \frac{e_{m_{2}}(w \zeta)}{w \zeta} d \zeta d w\right) \frac{\mathrm{ds}}{s} .
$$

So, $\mathcal{L}_{\delta+\frac{2 \pi j}{q}}$ are Stokes lines for $\widehat{u}(t, z)$, where $\delta=\theta=\arg z_{0}$ and $j=0, \ldots, q-1$. Moreover,

$$
\begin{aligned}
J_{\mathcal{L}_{\delta}} \widehat{u}_{j}(t, 0) & =\left[\frac{1}{2 \pi i} \oint_{|w|=\varepsilon} \frac{\varphi(w)}{w-\mathrm{se}^{\frac{2 \pi \mathrm{ij}}{q}}} d w\right]_{\theta}\left[\frac{e_{m}(s / t)}{\mathrm{qs}}\right]=\left[\varphi\left(\mathrm{se}^{\frac{2 \pi \mathrm{ij}}{q}}\right)\right]_{\theta}\left[\frac{e_{m}(s / t)}{\mathrm{qs}}\right] \\
& =\left\{\begin{array}{l}
{[\varphi(s)]_{\theta}\left[\frac{e_{m}(s / t)}{\mathrm{qs}}\right], \text { for } j=0} \\
0, \text { for } j=1,2, \ldots, q-1,
\end{array}\right.
\end{aligned}
$$

where $e_{m}$ is given by Eq. 20. Thus,

$$
J_{\mathcal{L}_{\delta}} \widehat{u}(t, 0)=\left[\sum_{j=0}^{q-1} \varphi\left(\mathrm{se}^{\frac{2 \pi \mathrm{ij}}{q}}\right)\right]_{\theta}\left[\frac{e_{m}(s / t)}{\mathrm{qs}}\right]=[\varphi(s)]_{\theta}\left[\frac{e_{m}(s / t)}{\mathrm{qs}}\right] .
$$


Case 4. In this part, we will study more general case, i.e.

$$
\left\{\begin{array}{l}
\partial_{m_{1}, t}^{p} u(t, z)=\partial_{m_{2}, z}^{q} u(t, z), \text { with } 0<\mathrm{ps}_{1}<\mathrm{qs}_{2}, \\
u(0, z)=\varphi(z), \\
\partial_{m_{1}, t}^{j} u(0, z)=0, \text { for } j=1,2, \ldots, p-1
\end{array}\right.
$$

with $\varphi(z) \in \mathcal{O}^{\frac{q}{\mathrm{qs}_{2}-\mathrm{ps}_{1}}}\left(\widetilde{\mathbb{C} \backslash\left\{z_{0}\right\}}\right)$ for some $z_{0} \in \mathbb{C} \backslash\{0\}$, where $m_{1}$ is a moment function of order $s_{1}>0$ corresponding to a kernel function $e_{m_{1}}(z)$ of order $1 / s_{1}, m_{2}$ is a moment function of order $s_{2}>0$ corresponding to a kernel function $e_{m_{2}}(z)$ of order $1 / s_{2}$. The above Cauchy problem has a formal solution

$$
\widehat{u}(t, z)=\sum_{n=0}^{\infty} \frac{\partial_{m_{2}, z}^{\mathrm{qn}} \varphi(z)}{m_{1}(\mathrm{pn})} t^{\mathrm{pn}} .
$$

By Theorem 2, $\mathcal{L}_{\delta+\frac{2 \pi j}{p}}$ are Stokes lines for $\widehat{u}(t, z)$, where $\delta=\frac{q}{p} \theta, \theta:=\arg z_{0}$ and $j=$ $0, \ldots, p-1$. To calculate the jumps across Stokes lines assume that $v(t, z):=u\left(t^{\frac{q}{p}}, z\right)$ and $\tilde{m}_{1}(n):=m_{1}\left(\frac{\mathrm{pn}}{q}\right)$ is a moment function of order $\frac{s_{1} p}{q}>0$ corresponding to a kernel function $e_{\tilde{m}_{1}}(z)=\frac{q}{p} e_{m_{1}}\left(z^{\frac{q}{p}}\right)$ of order $\frac{q}{s_{1} p}$. Then,

$$
\widehat{v}(t, z)=\sum_{n=0}^{\infty} \frac{\partial_{m_{2}, z}^{\mathrm{qn}} \varphi(z)}{\tilde{m}_{1}(\mathrm{qn})} t^{\mathrm{qn}}
$$

is a formal solution of the equation

$$
\left\{\begin{array}{l}
\partial_{\tilde{m}_{1}, t}^{q} v(t, z)=\partial_{m_{2}, z}^{q} v(t, z) \\
v(0, z)=\varphi(z) \in \mathcal{O}^{\frac{q}{\overline{q s}_{2}-\mathrm{ps}_{1}}}\left(\widetilde{\mathbb{C} \backslash\left\{z_{0}\right\}}\right) \\
\partial_{\tilde{m}_{1}, t}^{j} v(0, z)=0, \text { for } j=1,2, \ldots, q-1 .
\end{array}\right.
$$

Observe that $\widehat{u}(t, z)$ is a formal solution of the equation (21) if and only if $\widehat{v}(t, z)$ is a formal solution of the equation (22) (see also [15, Lemma 3]).

In this case, we reduce our problem to the one we considered in the case 3 . Thus, based on the obtained results, we have the following:

$$
v_{j}(t, z)=\int_{e^{\mathrm{id} \mathbb{R}_{+}}} e_{m}(s / t)\left(\frac{1}{2 \pi i} \oint_{|w|=\varepsilon} \varphi(w) \int_{0}^{\infty(\psi)} E_{m_{2}}\left(\mathrm{se}^{\frac{2 \pi \mathrm{ij}}{q}} \zeta\right) E_{m_{2}}(z \zeta) \frac{e_{m_{2}}(w \zeta)}{w \zeta} d \zeta d w\right) \frac{\mathrm{ds}}{s},
$$

so,

$$
\begin{aligned}
& J_{\mathcal{L}_{\theta}} \widehat{v}_{j}(t, 0)=\left[\frac{1}{2 \pi i} \oint_{|w|=\varepsilon} \frac{\varphi(w)}{w-\mathrm{se}^{\frac{2 \pi \mathrm{ij}}{q}}} d w\right]_{\theta}\left[\frac{e_{m}(s / t)}{\mathrm{qs}}\right]=\left[\varphi\left(s e^{\frac{2 \pi \mathrm{ij}}{q}}\right)\right]_{\theta}\left[\frac{e_{m}(s / t)}{\mathrm{qs}}\right] \\
& =\left\{\begin{array}{l}
{[\varphi(s)]_{\theta}\left[\frac{e_{m}(s / t)}{\mathrm{qs}}\right], \text { for } j=0} \\
0, \text { for } j=1,2, \ldots, q-1,
\end{array}\right.
\end{aligned}
$$

where $e_{m}$ is given by the following:

$$
e_{m}(u)=T_{\tilde{m}_{1}, d}^{-}\left(e_{m_{2}}(1 / z)\right)(1 / u)=-\frac{1}{2 \pi i} \int_{\gamma(d)} E_{\tilde{m}_{1}}\left(\frac{1}{u z}\right) e_{m_{2}}(1 / z) \frac{\mathrm{dz}}{z},
$$

with

$$
E_{\tilde{m}_{1}}\left(\frac{1}{\mathrm{uz}}\right)=\sum_{n=0}^{\infty} \frac{\left(\frac{1}{\mathrm{uz}}\right)^{n}}{\tilde{m}_{1}(n)}=\sum_{n=0}^{\infty} \frac{\left(\frac{1}{\mathrm{uz}}\right)^{n}}{m_{1}\left(\frac{\mathrm{pn}}{q}\right)}
$$


Thus

$$
J_{\mathcal{L}_{\theta}} \widehat{v}(t, 0)=\left[\sum_{j=0}^{q-1} \varphi\left(\mathrm{se}^{\frac{2 \pi \mathrm{ij}}{q}}\right)\right]_{\theta}\left[\frac{e_{m}(s / t)}{\mathrm{qs}}\right]=[\varphi(s)]_{\theta}\left[\frac{e_{m}(s / t)}{\mathrm{qs}}\right] .
$$

Hence

$$
J_{\mathcal{L}_{\delta}} \widehat{u}(t, 0)=J_{\mathcal{L}_{\theta}} \widehat{v}\left(t^{\frac{p}{q}}, 0\right)=[\varphi(s)]_{\theta}\left[\frac{e_{m}\left(s / t^{\frac{p}{q}}\right)}{\mathrm{qs}}\right] .
$$

Open Access This article is distributed under the terms of the Creative Commons Attribution 4.0 International License (http://creativecommons.org/licenses/by/4.0/), which permits unrestricted use, distribution, and reproduction in any medium, provided you give appropriate credit to the original author(s) and the source, provide a link to the Creative Commons license, and indicate if changes were made.

Publisher's Note Springer Nature remains neutral with regard to jurisdictional claims in published maps and institutional affiliations.

\section{References}

1. Balser W. From divergent power series to analytic functions, vol. 1582 of Lecture Notes in Mathematics. New York: Springer; 1994.

2. Balser W. Formal power series and linear systems of meromorphic ordinary differential equations. New York: Springer; 2000.

3. Balser W, Yoshino M. Gevrey order of formal power series solutions of inhomogeneous partial differential equations with constant coefficients. Funkcial Ekvac. 2010;53:411-434.

4. Hibino M. On the summability of divergent power series solutions for certain first-order linear PDEs. Opus Math. 2015;35:595-624.

5. Ichinobe K. On k-summability of formal solutions for a class of partial differential operators with time dependent coefficients. J Diff Equat. 2014;257:3048-3070.

6. Ichinobe K. On k-summability of formal solutions for certain higher order partial differential operators with polynomial coefficients. In: Analytic, algebraic and geometric aspects of differential equations, Trends in mathematics. Cham: Birkhäuser/Springer; 2017. p. 351-368.

7. Ichinobe K, Miyake M. On k-summability of formal solutions for certain partial differential operators with polynomial coefficients. Opus Math. 2015;35:625-653.

8. Immink G. Multisummability and the Stokes phenomenon. J Dyn Control Syst. 1995;1:483-534.

9. Kaneko A. Introduction to hyperfunctions, vol.3 of Mathematics and its applications. Dordrecht: Kluwer; 1988.

10. Köthe G. Dualität in der funktionentheorie. J Reine Angew Math. 1953;191:30-49.

11. Lastra A, Malek S, Sanz J. Summability in general Carleman ultraholomorphic classes. J Math Anal Appl. 2015;430:1175-1206.

12. Lastra A, Malek S, Sanz J. Strongly regular multi-level solutions of singularly perturbed linear partial differential equations. RM. 2016;70:581-614.

13. Malek S. On the Stokes phenomenon for holomorphic solutions of integro-differential equations with irregular singularity. J Dyn Control Syst. 2008;14:371-408.

14. Malgrange B, Ramis J-P. Fonctions multisommables. Ann Inst Fourier (Grenoble). 1992;42:353-368.

15. Michalik S. Analytic solutions of moment partial differential equations with constant coefficients. Funkcial Ekvac. 2013;56:19-50.

16. Michalik S. Summability of formal solutions of linear partial differential equations with divergent initial data. J Math Anal Appl. 2013;406:243-260.

17. Michalik S. Analytic summable solutions of inhomogeneous moment partial differential equations. Funkcial Ekvac. 2017;60:325-351.

18. Michalik S, Podhajecka B. The Stokes phenomenon for certain partial differential equations with meromorphic initial data. Asymptot Anal. 2016;99:163-182.

19. Remy P. Gevrey order and summability of formal series solutions of certain classes of inhomogeneous linear integro-differential equations with variable coefficients. J Dyn Control Syst. 2017;23:853-878.

20. Sternin BY, Shatalov VE. Borel-Laplace transform and asymptotic theory. Boca Raton: CRC Press; 1995. 
21. Tahara H, Yamazawa H. Multisummability of formal solutions to the Cauchy problem for some linear partial differential equations. J Diff Equat. 2013;255:3592-3637.

22. Tkacz B. The Stokes phenomenon for certain PDEs in a case when initial data have a finite set of singular points. In: Formal and analytic solutions of diff. equations, Springer proceedings in mathematics \& statistics. Cham: Springer; 2018. p. 79-93.

23. Yamazawa H, Yoshino M. Parametric Borel summability for some semilinear system of partial differential equations. Opus Math. 2015;35:825-845.

24. Yoshino M. Analytic continuation of Borel sum of formal solution of semilinear partial differential equation. Asymptot Anal. 2015;92:65-84.

25. Yoshino M. Parametric Borel summability of partial differential equations of irregular singular type. In: Analytic, algebraic and geometric aspects of differential equations, Trends in mathematics. Cham: Birkhäuser/Springer; 2017. p. 455-471. 